\title{
Anomaly Cancellation Condition in Lattice Gauge Theory
}

\author{
Hiroshi SUZUKI ${ }^{\star}$ \\ Department of Mathematical Sciences, Ibaraki University \\ Mito 310-8512, Japan ${ }^{\dagger}$ \\ and \\ The Abdus Salam International Center for Theoretical Physics, Trieste, Italy
}

\begin{abstract}
We study the gauge anomaly $\mathcal{A}$ defined on a 4 -dimensional infinite lattice while keeping the lattice spacing finite. We assume that (I) $\mathcal{A}$ depends smoothly and locally on the gauge potential, (II) $\mathcal{A}$ reproduces the gauge anomaly in the continuum theory in the classical continuum limit, and (III) $U(1)$ gauge anomalies have a topological property. It is then shown that the gauge anomaly $\mathcal{A}$ can always be removed by local counterterms to all orders in powers of the gauge potential, leaving possible breakings proportional to the anomaly in the continuum theory. This follows from an analysis of nontrivial local solutions to the Wess-Zumino consistency condition in lattice gauge theory. Our result is applicable to the lattice chiral gauge theory based on the Ginsparg-Wilson Dirac operator, when the gauge field is sufficiently weak $\|U(n, \mu)-1\|<\epsilon^{\prime}$, where $U(n, \mu)$ is the link variable and $\epsilon^{\prime}$ a certain small positive constant.
\end{abstract}

PACS numbers: 11.15.Ha, 11.30.Rd

Keywords: chiral gauge theory, lattice gauge theory

\footnotetext{
$\star$ E-mail: hsuzuki@ictp.trieste.it

$\dagger$ On leave of absence from.
} 


\section{Introduction}

If one puts Weyl fermions on a lattice while respecting desired physical properties, one has to sacrifice the $\gamma_{5}$-symmetry $[1,2]$. This implies that the gauge symmetry is inevitably broken on the lattice when Weyl fermions are coupled to the gauge field. This is rather natural, because we know that the gauge anomaly exists in the continuum theory [3-8]. However, even if the anomaly in the continuum theory cancels, $\operatorname{tr}_{R-L} T^{a}\left\{T^{b}, T^{c}\right\}=0\left[5^{-}\right.$ $8]$, the fermion determinant is not gauge invariant in general when the lattice spacing is finite, $a \neq 0$. Then the gauge degrees of freedom do not decouple and it becomes quite unclear whether properties of the continuum theory (such as unitarity) are reproduced in the continuum limit, after the effect of dynamical gauge fields is taken into account. Basically this is the origin of difficulties of chiral gauge theories on the lattice [9]. It is thus quite important to understand the structure of breakings of the gauge symmetry on the lattice, which will be denoted by $\mathcal{A}$, while keeping the lattice spacing finite.

What is the possible structure of $\mathcal{A}$ for $a \neq 0$ ? This question appears meaningless unless certain conditions are imposed on $\mathcal{A}$. After all, uniqueness of the gauge anomaly in the continuum theory $[5-8,10-17]$ is lost for a finite ultraviolet cutoff, and the explicit form of the breaking $\mathcal{A}$ is expected to depend strongly on the details of the lattice formulation. But what kind of conditions can strongly constrain the structure of $\mathcal{A}$ ? And, under such conditions, is it possible to relate $\mathcal{A}$ and the anomaly in the continuum theory? It seemed almost impossible to answer these questions. (This statement is not completely true: If one restricts operators with the mass dimension $\leq 5$ (we assign one mass dimension to the ghost field), the complete classification of possible breakings has been known in the context of the Rome approach [18].)

The atmosphere has changed after Lüscher's theorem on the $\gamma_{5}$-anomaly in the abelian lattice gauge theory $G=U(1)$ appeared [19]. Assuming smoothness, locality ${ }^{\ddagger}$ and the topological nature of the anomaly, he proved the theorem for a 4-dimensional infinite lattice,

$\ddagger$ The meaning of the locality is of course different from that of the continuum theory. We will explain this terminology in detail in the next section. 
which corresponds to $§$

$$
\begin{aligned}
\mathcal{A} & =\delta_{B} \ln \operatorname{Det} M^{\prime}[A] \\
& =\sum_{n} c(n)\left[\alpha+\beta_{\mu \nu} F_{\mu \nu}(n)+\gamma \varepsilon_{\mu \nu \rho \sigma} F_{\mu \nu}(n) F_{\rho \sigma}(n+\widehat{\mu}+\widehat{\nu})+\Delta_{\mu}^{*} k_{\mu}(n)\right]
\end{aligned}
$$

where Det $M^{\prime}$ is a fermion determinant and $\delta_{B}$ is the BRS transformation [10] corresponding to the gauge transformation in the abelian lattice gauge theory, $\delta_{B} A_{\mu}(n)=\Delta_{\mu} c(n)$ and $\delta_{B} c(n)=0 ; c(n)$ stands for the abelian Faddeev-Popov ghost field defined on the lattice. In eq. (1.1), $\alpha, \beta_{\mu \nu}$ and $\gamma$ are constants, and $k_{\mu}(n)$ in the last term is a local and gauge invariant current. Note that eq. (1.1) holds for finite lattice spacing and that the structure is quite independent of the details of the formulation. In this sense, this theorem provides a universal characterization of the gauge anomaly in abelian lattice chiral gauge theory. Moreover, the theorem asserts that the anomaly cancellation in the abelian lattice theory is (almost) equivalent to that of the continuum theory: The first two constants vanish, $\alpha=\beta_{\mu \nu}=0$, if the anomaly is a pseudoscalar quantity. The term proportional to $\gamma$ is cancelled if $\sum_{R} e_{R}^{3}-\sum_{L} e_{L}^{3}=0$. Here $e_{H}$ stands for the $U(1)$ charge, because we have absorbed the $U(1)$ charge in $c$ and in $F_{\mu \nu}$. Finally, the last term of the breaking (1.1) can be removed by adding the local counterterm $\mathcal{B}=\sum_{n} A_{\mu}(n) k_{\mu}(n)$ to the effective action $\ln$ Det $M^{\prime} \rightarrow \ln$ Det $M^{\prime}+\mathcal{B}$, because $\delta_{B} \mathcal{B}=\sum_{n} \Delta_{\mu} c(n) k_{\mu}(n)=-\sum_{n} c(n) \Delta_{\mu}^{*} k_{\mu}(n)$. This argument shows that the effective action with finite lattice spacing can be made gauge invariant if (and only if) the fermion multiplet is anomaly-free! This remarkable observation was fully utilized in the existence proof of an exactly gauge invariant lattice formulation of anomaly-free abelian chiral gauge theories [20].

In this paper, we attempt to generalize the above theorem (1.1) for general (compact) gauge groups. Our scheme is somewhat different from that of refs. [19,21]. In ref. [21], this problem in nonabelian theories was shown to be equivalent to a classification of gauge invariant topological fields in (4+2)-dimensional space, where 4 dimensions are discrete and 2 dimensions are continuous. Instead, in this paper, we analyze general nontrivial local solutions to the Wess-Zumino consistency condition [22] in lattice gauge theory. For a generic

$\S$ For our notation, see appendix A. 
gauge group, the BRS transformation is defined by:

$$
\delta_{B} U(n, \mu)=U(n, \mu) c(n+\widehat{\mu})-c(n) U(n, \mu), \quad \delta_{B} c(n)=-c(n)^{2} .
$$

Since this BRS transformation is nilpotent $\delta_{B}^{2}=0$, the breaking $\mathcal{A}=\delta_{B} \ln$ Det $M^{\prime}$ must satisfy the Wess-Zumino consistency condition

$$
\delta_{B} \mathcal{A}=0
$$

like in the continuum theory $[22,10]$. In the continuum theory, consistency and uniqueness of anomaly-free chiral gauge theories on the perturbative level follow from detailed analyses of the consistency condition [10-17] (for a more complete list of references, see ref. [17]). We will see below that the consistency condition (1.3), combined with the locality in the sense of ref. [19], strongly constrains the possible structure of $\mathcal{A}$, as it does in the continuum theory. Our basic strategy is to imitate as much as possible the procedure in the continuum theory, especially that of ref. [16]. Of course, there are many crucial differences between continuum and lattice theories and how to handle these differences becomes the key to our "algebraic" approach.

The organization of this paper is as follows. Our main theorems which generalize eq. (1.1) are stated in section 3. Our theorems are applicable only if the gauge anomaly $\mathcal{A}$ depends locally on the gauge field. The only framework known at present, which possesses this property is the formulation of refs. [20,21] based on the Ginsparg-Wilson Dirac operator [23$25]$, or equivalently the overlap formulation [26,27]. Therefore, in section 2, we summarize basic properties of the gauge anomaly along the formulation of ref. [21]. At the same time, we introduce notions of admissibility and of locality. We also introduce the gauge potential and define the so-called "perturbative configuration." Sections 4 to 6 are entirely devoted to the determination of general nontrivial local solutions to the consistency condition in the abelian theory $G=U(1)^{N}$. In section 4 , we give some preliminaries. In section 5 , we prove several lemmas concerning cohomology on an infinite lattice. Here the technique of noncommutative differential calculus [28-31] turns out to be a powerful tool [32,33]. Utilizing these lemmas,

I This transformation is obtained by parameterizing the gauge transformation parameter in $U(n, \mu) \rightarrow$ $g(n)^{-1} U(n, \mu) g(n+\widehat{\mu})$ by $g=\exp (\lambda c)$ where $\lambda$ stands for an infinitesimal Grassmann parameter. 
in section 6, we first determine a complete list of nontrivial local solutions to the consistency condition in the abelian theory. Here the ghost number of the solution is arbitrary. Then we restrict the ghost number of the solution to unity. After imposing several conditions, we obtain the content of the theorem for the abelian theory. Section 7 is devoted to the nonabelian extension. In section 7.1, we derive a basic lemma which guarantees the adjoint invariance of nontrivial solutions. In section 7.2, by using several assumptions, we show the uniqueness of the nontrivial local anomaly to all orders in powers of the gauge potential. This establishes the content of our theorem for nonabelian theories, which will be stated in section 3. In section 7.3, we explicitly write down such a nontrivial local anomaly by utilizing the interpolation technique of lattice fields [34,35]. The last section is devoted to concluding remarks. Our notation is summarized in appendix A. In appendix B, we explain the calculation of the Wilson line which appears in the integrability condition of ref. [21].

\section{Gauge anomaly in the Ginsparg-Wilson approach}

\subsection{Admissibility, LOCAlity and the GaUge potential}

The "admissible" gauge field is defined by [21]

$$
\|P(n, \mu, \nu)-1\|<\epsilon, \quad \text { for all } n, \mu, \nu,
$$

where $P(n, \mu, \nu)$ is the plaquette variable in the representation to which the Weyl fermion belongs and $\epsilon$ is a certain small positive constant. In this expression, $\|\mathcal{O}\|$ is the operator norm defined by [36]

$$
\|\mathcal{O}\|=\sup _{v \neq 0} \frac{\|\mathcal{O} v\|}{\|v\|}
$$

where the norm on the right hand side is defined by the standard norm for vectors. The reason for this restriction of field space is two-fold:

Consider a finite lattice. Let us suppose that the Dirac operator satisfies an index theorem. Namely, a difference of numbers of normalizable zero modes of the Dirac operator with opposite chirality is equal to the topological charge of the gauge field configuration. The index is an integer and thus inevitably jumps even if the gauge field configuration changes

smoothly. This argument suggests that such a Dirac operator cannot be a smooth function 
of the gauge field. Smoothness of the Dirac operator and in turn that of the gauge anomaly are thus expected to hold only within a restricted field space. In fact, a detailed analysis [37] of Neuberger's overlap Dirac operator [25], which satisfies the index theorem [38,39], shows that the Dirac operator depends smoothly and locally on the gauge field when $\epsilon \leq 1 / 30$ in eq. (2.1). Our proof is valid only when the gauge anomaly depends on the gauge field smoothly and locally.

Closely related to the above point, we note that any configuration of the lattice gauge field can smoothly be deformed into the trivial one, $U(n, \mu)=1$, and thus the topology of the gauge field space is trivial if no restriction is imposed. On the other hand, it has been known [40] that, under the condition (2.1), one can define a nontrivial principal bundle over a periodic lattice such that the field space is divided into topological sectors. For example, for the fundamental representation of $S U(2), \epsilon \leq 0.015$ is enough for the construction of ref. [40] to work. Later we will utilize the interpolation method of ref. [34] which is based on the section of the principal bundle of ref. [40].

Note that eq. (2.1) is a gauge invariant condition. The gauge transformed configurations of an admissible configuration are all admissible. However, the structure of the space of admissible configurations is quite complicated, and no simple parameterization in terms of the gauge potential has been known except for abelian cases [19]. This is the reason why our theorem for nonabelian theories is in practice applicable only for the "perturbative configurations" which will be explained below.

As noted in the introduction, our basic strategy is to imitate the argument in the continuum theory. The first important difference from the continuum theory is the notion of locality. The anomaly is a local quantity when the ultraviolet cutoff is sent to infinity. But of course this is not the case for $a \neq 0$ so that we need an appropriate notion which works on the lattice. Here we follow the definition of ref. [19] (see also ref. [41]). Suppose that $\phi(n)$ is a field on the lattice which depends on link variables $U$. The field $\phi(n)$ may depend on the link variable $U(m, \mu)$ at a distant link from the site $n$. We say that $\phi(n)$ locally depends on the link variable, if this dependence on $U(m, \mu)$ becomes exponentially weak as $|n-m| \rightarrow \infty$. To be more precise, consider the following decomposition:

$$
\phi(n)=\sum_{k=1}^{\infty} \phi_{k}(n)
$$


where $\phi_{k}(n)$ depends only on link variables $U$ inside a block of size $k$ centered at the site $n$ (such a field $\phi_{k}(n)$ is called ultra-local). If all these fields $\phi_{k}(n)$ and their derivatives $\phi_{k}\left(n ; m_{1}, \mu_{1} ; \cdots ; m_{N}, \mu_{N}\right)$ with respect to the link variables $U\left(m_{1}, \mu_{1}\right), \ldots, U\left(m_{N}, \mu_{N}\right)$ are bounded as

$$
\left|\phi_{k}\left(n ; m_{1}, \mu_{1} ; \cdots ; m_{N}, \mu_{N}\right)\right| \leq C_{N} k^{p_{N}} \exp (-\theta k)
$$

by the constants $C_{N}, p_{N}$ and $\theta$, which are all independent of link variable configurations, then we say that $\phi(n)$ locally depends on the link variable. In what follows, we also introduce the gauge potential and the ghost field. The same terminology will be used by simply replacing "link variable" by the name of each field. When no confusion arises, we say simply that $\phi(n)$ is local. Also when a functional is given by a sum of such local fields, $\Phi=\sum_{n} \phi(n)$, we simply say that $\Phi$ is local. If $\phi(n)$ is a local field, the effective range of dependences is a finite number in lattice units. Physically, therefore, this locality can be regarded as equivalent to ultra-locality. The technical reason for this definition of locality is that the Dirac operator which satisfies the Ginsparg-Wilson relation cannot be ultra-local in general $[42,43]$ and, on the other hand, we can apply the Poincaré lemma of ref. [19] if dependences are exponentially suppressed.

The basic degrees of freedom in lattice gauge theory are link variables. However we prefer to stick to the gauge potential, because its use is essential for arguments in the continuum theory. To stretch the validity of our argument as far as possible, we consider the following two cases.

Case I. When the gauge group is abelian $G=U(1)^{N}$. If we take $0<\epsilon \leq 1$ in eq. (2.1) or equivalently (the superscript $a$ here labels each $U(1)$ factor in $G$ )

$$
\left\|\operatorname{Ln} P^{a}(n, \mu, \nu)\right\|<\frac{\pi}{3}, \quad \text { for all } a, n, \mu, \nu
$$

there exists a relatively simple prescription [19] which allows a complete parameterization of the space of admissible gauge fields. Under the condition (2.5), one can associate the abelian gauge potential to the link variable such that

$$
U^{a}(n, \mu)=\exp A_{\mu}^{a}(n), \quad-\infty<\frac{1}{i} A_{\mu}^{a}(n)<\infty
$$


and moreover

$$
\operatorname{Ln} P^{a}(n, \mu, \nu)=\Delta_{\mu} A_{\nu}^{a}(n)-\Delta_{\nu} A_{\mu}^{a}(n) .
$$

From this relation and eq. (2.5), one concludes that if a configuration $A_{\mu}^{a}$ is admissible, the rescaled one $t A_{\mu}^{a}$ with $0 \leq t \leq 1$ is also admissible. In this prescription [19] (a closely related prescription for 2-dimensional periodic lattices was first given in ref. [35]), the abelian gauge potential $A_{\mu}^{a}(n)$ corresponding to the given link variables $U^{a}(n, \mu)$ is not unique. Also this mapping does not preserve the locality. Nevertheless, as far as gauge invariant quantities are concerned, such an ambiguity disappears and the locality becomes common for both variables. See refs. $[19,44]$ for details.

Case II. For a general (compact) gauge group $G$, we define

$$
U(n, \mu)=\exp A_{\mu}(n), \quad\left\|A_{\mu}(n)\right\| \leq \pi
$$

and we further impose

$$
\left\|A_{\mu}(n)\right\|<\frac{1}{4} \ln (1+\epsilon) \leq \pi, \quad \text { for all } \mu \text { and } n
$$

By noting $\left\|\mathcal{O}+\mathcal{O}^{\prime}\right\| \leq\|\mathcal{O}\|+\left\|\mathcal{O}^{\prime}\right\|$ and $\left\|\mathcal{O O}^{\prime}\right\| \leq\|\mathcal{O}\|\left\|\mathcal{O}^{\prime}\right\|[36]$, one can see that configurations which satisfy eq. (2.9) are in fact admissible, i.e., they satisfy eq. (2.1). Note that eq. (2.9) is a very restrictive condition and contains only a small portion of admissible configurations; in fact, the condition (2.9) is not gauge invariant. We call configurations which satisfy eq. (2.9) "perturbative." For perturbative configurations, all link variables are close to unity $\|U(n, \mu)-1\|<\epsilon^{\prime}=(1+\epsilon)^{1 / 4}-1$. Unfortunately, our theorem for nonabelian theories is applicable only for this restricted space, when the admissibility (2.1) is required.

\subsection{Fermion Determinant and the gauge Anomaly}

In this subsection, we study basic properties of the gauge anomaly appearing in the formulation based on the Ginsparg-Wilson Dirac operator [20,21] with a particular choice of the integration measure. As noted sometimes $[45,46]$, this formulation can be reinterpreted in terms of the overlap formulation [26,27]. Therefore it must be possible to repeat a similar argument also in the context of the overlap formulation. 
Following refs. $[20,21]$, we define the fermion determinant as

$$
\operatorname{Det} M^{\prime}=\int d[\psi] d[\bar{\psi}] \exp \left[-\sum_{n} \bar{\psi}(n) D \psi(n)\right], \quad \widehat{P}_{H} \psi(n)=\psi(n),
$$

where the Dirac operator $D$ satisfies the Ginsparg-Wilson relation $\gamma_{5} D+D \gamma_{5}=D \gamma_{5} D$ [23]. We assume that the Dirac operator $D$ is gauge covariant and local in the sense of ref. [20] and depends smoothly on the gauge field. Thus we assume the admissibility (2.1) for gauge field configurations. The chirality of the fermion is defined with respect to the Ginsparg-Wilson chiral matrix $\widehat{\gamma}_{5}=\gamma_{5}(1-D)[47,41,48]$. Namely, the projection operator has been defined by $\widehat{P}_{H}=\left(1+\epsilon_{H} \widehat{\gamma}_{5}\right) / 2$. The chirality of the anti-fermion is, on the other hand, defined by the conventional $\gamma_{5}$ matrix.

The integration measure for the fermion $d[\psi]$ in eq. (2.10) thus depends on the gauge field nontrivially due to the condition $\widehat{P}_{H} \psi=\psi$. However this condition alone does not specify the integration measure uniquely. For definiteness, we make the following choice which starts with the particular "measure term" [49]

$$
\mathcal{L}_{\eta}^{\prime}=-i \epsilon_{H} \int_{0}^{1} d s \operatorname{Tr} \widehat{P}_{H}\left[\partial_{s} \widehat{P}_{H}, \delta_{\eta} \widehat{P}_{H}\right]
$$

where Tr stands for the summation over lattice points $\sum_{n}$ of the diagonal $(n, n)$ components as well as traces over the gauge and the spinor indices. In the above expression, $\eta$ stands for the infinitesimal variation of link variables

$$
\delta_{\eta} U(n, \mu)=\eta_{\mu}(n) U(n, \mu)
$$

We have to specify the $s$-dependence in eq. (2.11). As a simple choice, we take

$$
U(n, s, \mu)=\exp \left[s A_{\mu}(n)\right], \quad 0 \leq s \leq 1
$$

for both cases I (2.6) and II (2.8) above. Note that the line in the configuration space $U(n, s, \mu)$ which connects 1 and $U(n, \mu)$ is contained in the admissible space (2.1) and, for the case II, in the perturbative region (2.9). The functional (2.11) depends smoothly and

\footnotetext{
$\star \mathcal{L}_{\eta}^{\prime}$ identically vanishes when the representation of the Weyl fermion is (pseudo-)real [49].
} 
locally on the gauge potential due to the assumed properties of the Dirac operator $\left(\mathcal{L}_{\eta}^{\prime}\right.$ does not contain the inverse of the Dirac operator). Since the functional $\mathcal{L}_{\eta}^{\prime}$ is linear in $\eta_{\mu}$, it may be written as

$$
\mathcal{L}_{\eta}^{\prime}=\sum_{n} \eta_{\mu}^{a}(n) j_{\mu}^{\prime a}(n), \quad \eta_{\mu}(n)=\eta_{\mu}^{a}(n) T^{a} .
$$

This current $j_{\mu}^{\prime a}$ depends smoothly and locally on the gauge potential.

Now, using the Ginsparg-Wilson relation, one can show [49] that $\mathcal{L}_{\eta}^{\prime}$ satisfies the differential form of the integrability condition $[20,21]:^{\dagger}$

$$
\delta_{\eta} \mathcal{L}_{\zeta}^{\prime}-\delta_{\zeta} \mathcal{L}_{\eta}^{\prime}+\mathcal{L}_{[\eta, \zeta]}^{\prime}=-i \epsilon_{H} \operatorname{Tr} \widehat{P}_{H}\left[\delta_{\eta} \widehat{P}_{H}, \delta_{\zeta} \widehat{P}_{H}\right]
$$

Moreover, by considering a one-parameter family of gauge fields, $U_{t}(n, \mu)(0 \leq t \leq 1)$ and introducing the transporting operator $Q_{t}$ by [21]

$$
\partial_{t} Q_{t}=\left[\partial_{t} P_{t}, P_{t}\right] Q_{t}, \quad P_{t}=\left.\widehat{P}_{H}\right|_{U \rightarrow U_{t}}, \quad Q_{0}=1,
$$

one can show (appendix B) that $\mathcal{L}_{\eta}^{\prime}$ satisfies the integrability in the integrated form [21] for an arbitrary closed loop $U_{0}(n, \mu)=U_{1}(n, \mu)$ (here $\left.\eta_{\mu}(n)=\partial_{t} U_{t}(n, \mu) U_{t}(n, \mu)^{-1}\right)$

$$
W^{\prime}=\exp \left(i \int_{0}^{1} d t \mathcal{L}_{\eta}^{\prime}\right)=\operatorname{Det}\left(1-P_{0}+P_{0} Q_{1}\right)^{-\epsilon_{H}},
$$

as long as the loop $U_{t}(n, \mu)$ is contained within the perturbative region (2.9) for the case II. Since both the space of admissible fields (2.5) for case I and the space of perturbative configurations (2.9) for case II are contractable, there is no global obstruction [50] which is a lattice counterpart of the Witten's anomaly [51]. Eq. (2.17) guarantees that there exists an integration measure $d[\psi] d[\bar{\psi}]$ which corresponds to the measure term $\mathcal{L}_{\eta}^{\prime}$ [21]. In particular, the infinitesimal variation of the fermion determinant (2.10) is given by

$$
\delta_{\eta} \ln \operatorname{Det} M^{\prime}=\operatorname{Tr} \delta_{\eta} D \widehat{P}_{H} D^{-1}+i \epsilon_{H} \mathcal{L}_{\eta}^{\prime}
$$

We have completely specified the fermion determinant (2.10) up to a physically irrelevant proportionality constant. This fermion determinant is, however, not gauge invariant in

$\dagger$ Here we assume that $\eta$ and $\zeta$ are independent of the gauge field. 
general. The resulting gauge anomaly $\mathcal{A}=\delta_{B} \ln$ Det $M^{\prime}$ is obtained simply by setting

$$
\eta_{\mu}(n)=U(n, \mu) c(n+\widehat{\mu}) U(n, \mu)^{-1}-c(n)
$$

in eq. (2.12). Then from eqs. (2.18) and (2.14), we have

$$
\mathcal{A}=\epsilon_{H} \operatorname{Tr} c \gamma_{5}\left(1-\frac{1}{2} D\right)-i \epsilon_{H} \sum_{n} c^{a}(n)\left[j_{\mu}^{\prime}(n)-U(n-\widehat{\mu}, \mu)^{-1} j_{\mu}^{\prime}(n-\widehat{\mu}) U(n-\widehat{\mu}, \mu)\right]^{a},
$$

where use of the gauge covariance $\delta_{B} D=[D, c]$ for $s=1$ and the Ginsparg-Wilson relation has been made. Manifestly, this anomaly $\mathcal{A}$ depends smoothly and locally on the gauge potential (and on the ghost field) from the assumed properties of the Dirac operator and from the properties of the current $j_{\mu}^{\prime a}$.

Let us next study the classical continuum limit of $\mathcal{A}$. The gauge potential in the classical continuum limit $A_{\mu}(x)$ is introduced by the conventional manner

$$
U(n, \mu)=\mathcal{P} \exp \left[a \int_{0}^{1} d u A_{\mu}(n+(1-u) \widehat{\mu} a)\right],
$$

where $\mathcal{P}$ stands for the path-ordered product. Then the first term of eq. (2.20) produces the covariant gauge anomaly which can be deduced from the general arguments [52,21] or from explicit calculations using Neuberger's overlap Dirac operator [53-55] as ${ }^{\ddagger}$

$$
\epsilon_{H} \operatorname{Tr} c \gamma_{5}\left(1-\frac{1}{2} D\right) \stackrel{a \rightarrow 0}{\rightarrow}-\frac{\epsilon_{H}}{8 \pi^{2}} \int d^{4} x \varepsilon_{\mu \nu \rho \sigma} \operatorname{tr} c \partial_{\mu}\left(A_{\nu} \partial_{\rho} A_{\sigma}+\frac{2}{3} A_{\nu} A_{\rho} A_{\sigma}\right),
$$

for a single Weyl fermion. For the second term of eq. (2.20), which corresponds to a divergence of the so-called Bardeen-Zumino current [56] in the continuum theory, it is easier to consider $\mathcal{L}_{\eta}^{\prime}$ (2.11) instead of the divergence of the current $j_{\mu}^{\prime a}$. With the choice (2.13), we see in the classical continuum limit ${ }^{\S}$

$$
\delta_{B} \widehat{P}_{H}=s\left[\widehat{P}_{H}, c\right]+O(a) .
$$

Then by using the Ginsparg-Wilson relation, we have

$$
i \epsilon_{H} \mathcal{L}_{\eta}^{\prime}=-\frac{\epsilon_{H}}{2} \int_{0}^{1} d s s \partial_{s} \operatorname{Tr} c \gamma_{5}(1-D)+O(a) .
$$

It is possible to argue that the $O(a)$-term in eq. (2.23) contributes only to the $O(a)$-term

$\ddagger$ Of course, we assume that parameters in the Dirac operator has been chosen such that there is only one massless degree of freedom.

$\S$ For abelian cases, the relation $\delta_{B} \widehat{P}_{H}=s\left[\widehat{P}_{H}, c\right]$ holds for arbitrary $a$. 
in eq. (2.24) from the mass dimension and the pseudoscalar nature of $i \epsilon_{H} \mathcal{L}_{\eta}^{\prime}$ (assuming that Lorentz invariance is restored in the classical continuum limit). Then, from eq. (2.22) with the substitution $A_{\mu} \rightarrow s A_{\mu}$, we have

$$
i \epsilon_{H} \mathcal{L}_{\eta}^{\prime} \stackrel{a \rightarrow 0}{\rightarrow} \frac{\epsilon_{H}}{8 \pi^{2}} \int d^{4} x \varepsilon_{\mu \nu \rho \sigma} \operatorname{tr} c \partial_{\mu}\left(\frac{2}{3} A_{\nu} \partial_{\rho} A_{\sigma}+\frac{1}{2} A_{\nu} A_{\rho} A_{\sigma}\right)
$$

Combining eqs. (2.22) and (2.25), we have the correct consistent anomaly in the continuum theory:

$$
\mathcal{A} \stackrel{a \rightarrow 0}{\rightarrow}-\frac{\epsilon_{H}}{24 \pi^{2}} \int d^{4} x \varepsilon_{\mu \nu \rho \sigma} \operatorname{tr} c \partial_{\mu}\left(A_{\nu} \partial_{\rho} A_{\sigma}+\frac{1}{2} A_{\nu} A_{\rho} A_{\sigma}\right) .
$$

This expression is for a simple gauge group. The gauge anomaly for a generic gauge group $G=\prod_{\alpha} G_{\alpha}$ can be obtained by simply substituting $c \rightarrow \sum_{\alpha} c^{G_{\alpha}}$ and $A_{\mu} \rightarrow \sum_{\alpha} A_{\mu}^{G_{\alpha}}$ in eq. (2.26). To have the standard form of the anomaly for $G=\prod_{\alpha} G_{\alpha}$, we add the local counterterm to the effective action, $\ln \operatorname{Det} M^{\prime \prime}=\ln \operatorname{Det} M^{\prime}+\mathcal{S}$, or to the measure term $i \epsilon_{H} \mathcal{L}^{\prime \prime}=i \epsilon_{H} \mathcal{L}^{\prime}+\delta_{\eta} \mathcal{S}$, where

$$
\mathcal{S}=\frac{\epsilon_{H}}{144 \pi^{2}} \sum_{n} \varepsilon_{\mu \nu \rho \sigma} V(n, \mu)^{U(1)_{\beta}} \operatorname{tr} V(n, \nu)^{(\alpha)} V(n, \rho)^{(\alpha)} V(n, \sigma)^{(\alpha)}
$$

and $V(n, \mu)=\left[U(n, \mu)-U(n, \mu)^{\dagger}\right] / 2$. The superscript $\alpha$ runs over simple groups in $G$, and $\beta$ denotes each $U(1)$ factor in $G$. $\mathcal{S}$ depends smoothly and locally on the link variable and the modification does not affect the integrability, eqs. (2.15) and $(2.17){ }^{*}$ The counterterm $\mathcal{S}$ was chosen such that its classical continuum limit becomes $\epsilon_{H} \int d^{4} x \varepsilon_{\mu \nu \rho \sigma} A_{\mu}^{U(1)_{\beta}} \operatorname{tr} A_{\nu}^{(\alpha)} A_{\rho}^{(\alpha)} A_{\sigma}^{(\alpha)} /\left(144 \pi^{2}\right)$. Then the gauge anomaly of the modified effective action becomes

$$
\begin{aligned}
\mathcal{A}^{a \rightarrow 0} \rightarrow-\frac{\epsilon_{H}}{24 \pi^{2}} \int d^{4} x\{ & \varepsilon_{\mu \nu \rho \sigma} \operatorname{tr} c^{(\alpha)} \partial_{\mu}\left[A_{\nu}^{(\alpha)} \partial_{\rho} A_{\sigma}^{(\alpha)}+\frac{1}{2} A_{\nu}^{(\alpha)} A_{\rho}^{(\alpha)} A_{\sigma}^{(\alpha)}\right] \\
& +\varepsilon_{\mu \nu \rho \sigma} c^{U(1)_{\beta}} \partial_{\mu} A_{\nu}^{U(1)_{\beta}} \partial_{\rho} A_{\sigma}^{U(1)_{\beta}} \\
& +\varepsilon_{\mu \nu \rho \sigma} c^{U(1)_{\beta}} \operatorname{tr} \partial_{\mu}\left[A_{\nu}^{(\alpha)} \partial_{\rho} A_{\sigma}^{(\alpha)}+\frac{2}{3} A_{\nu}^{(\alpha)} A_{\rho}^{(\alpha)} A_{\sigma}^{(\alpha)}\right] \\
& \left.+2 \varepsilon_{\mu \nu \rho \sigma} \operatorname{tr}\left[c^{(\alpha)} \partial_{\mu} A_{\nu}^{(\alpha)}\right] \partial_{\rho} A_{\sigma}^{U(1)_{\beta}}\right\}
\end{aligned}
$$

I Strictly speaking, an explicit calculation of eq. (2.11) or of eq. (2.24) in the classical continuum limit, using, say, Neuberger's overlap Dirac operator, has not been carried out in the literature. A corresponding calculation on the linearized level in the overlap formulation was given in the last reference of ref. [27]. See also ref. [21]

* Note that (provided that $\eta$ and $\zeta$ are independent of the gauge field) the relation $\left(\delta_{\eta} \delta_{\zeta}-\delta_{\zeta} \delta_{\eta}+\delta_{[\eta, \zeta]}\right) \mathcal{S}=$ 0 holds for any functional $\mathcal{S}$ of the link variable. 
in the classical continuum limit.

Finally, we have to mention a topological property of the gauge anomaly which is associated with each $U(1)$ factor. Going back to the infinitesimal variation (2.18) for $a \neq 0$, the gauge anomaly is obtained by substituing $\delta_{\eta} \rightarrow \delta_{B}$. Since, as already noted,

$$
\delta_{B} \widehat{P}_{H}=s\left[\widehat{P}_{H}, c^{U(1)_{\beta}}\right]+\left(\text { terms proportional to } c^{(\alpha)}\right),
$$

where $\alpha$ stands for simple groups, we have [49] after using the Ginsparg-Wilson relation

$$
\mathcal{A}=\epsilon_{H} \int_{0}^{1} d s \operatorname{Tr} c^{U(1)_{\beta}} \gamma_{5}\left(1-\frac{1}{2} D\right)+\left(\text { terms proportional to } c^{(\alpha)}\right) .
$$

The combination $q=\operatorname{tr} \gamma_{5}(1-D / 2)$ appearing here is a topological field $[38,39]$ such that

$$
\sum_{n} \delta q(n)=0
$$

for an arbitrary local variation of the gauge field $\delta$. Therefore we see that

$$
\delta \mathcal{A}=\left(\text { terms proportional to } c^{(\alpha)}\right), \quad \text { for } \quad c^{U(1)_{\beta}}(n) \rightarrow \text { const. }
$$

where $\delta$ is an arbitrary local variation of the gauge potential. Note that, since $\delta_{B} A_{\mu} \rightarrow$ (terms prop. to $c^{(\alpha)}$ ) for $c^{U(1)_{\beta}}(n) \rightarrow$ const., this topological property for abelian factors holds even after the addition of local terms to the effective action, such as $\mathcal{S}$ in eq. (2.27).

We have thus observed that (I) the anomaly on the lattice $\mathcal{A}=\delta_{B} \ln \operatorname{Det} M^{\prime \prime}$ depends smoothly and locally on the gauge potential and on the ghost field, (II) $\mathcal{A}$ reproduces the gauge anomaly in the continuum theory in the classical continuum limit as in eq. (2.28), and (III) $U(1)$ gauge anomalies in $\mathcal{A}$ have the topological property (2.32). In the following sections, we show that such an anomaly $\mathcal{A}=\delta_{B} \ln$ Det $M^{\prime \prime}$ on an infinite lattice can always be written as $\mathcal{A}=\delta_{B} \mathcal{B}$, where $\mathcal{B}$ depends smoothly and locally on the gauge potential, if (and only if) the anomaly in the continuum theory is canceled, $\operatorname{tr}_{R-L} T^{a}\left\{T^{b}, T^{c}\right\}=0$ etc. This statement holds to all orders in powers of the gauge potential (for nonabelian cases). This 
implies that, for an anomaly-free fermion multiplet, one can improve the fermion determinant according to

$$
\operatorname{Det} M^{\prime \prime}[A] \rightarrow \operatorname{Det} M[A]=\operatorname{Det} M^{\prime \prime}[A] \exp (-\mathcal{B}[A])
$$

so that the improved fermion determinant Det $M[A]$ has the exact gauge invariance.* Therefore, to all orders of the gauge potential, there exists a gauge invariant lattice formulation of anomaly-free nonabelian chiral gauge theories, as far as the perturbative configurations (2.9) on an infinite lattice are concerned.

In the context of the overlap formulation, our choice of the measure term (2.11) corresponds to a particular choice of the phase of the vacuum state. The formula corresponding to eq. (2.20) was given in the last reference of ref. [27]. The gauge anomaly and Witten's anomaly as local and global obstructions in the overlap formulation were studied in detail in ref. [57]. See also ref. [46].

\section{Results}

In this section, we present our main results in a summarized form. For the abelian gauge group $G=U(1)^{N}$, we will show the following theorem.

Theorem (Abelian theory). Let $\mathcal{A}[c, A]$ be the gauge anomaly defined on a 4-dimensional infinite hypercubic lattice. Suppose that (I) $\mathcal{A}$ depends smoothly and locally on the abelian gauge potential $A_{\mu}^{a}$ and on the abelian ghost field $c^{a}$, (II) $\mathcal{A}$ reproduces for smooth field configurations the gauge anomaly in the continuum theory (2.28) in the classical continuum limit, and (III) $\mathcal{A}$ has the topological property

$$
\delta \mathcal{A}=0, \quad \text { for } \quad c^{a}(n) \rightarrow \text { const. }
$$

where $\delta$ is an arbitrary local variation of the gauge potential. Then $\mathcal{A}$ is always of the form (for a single Weyl fermion)

$$
\mathcal{A}[c, A]=-\frac{\epsilon_{H}}{96 \pi^{2}} \sum_{n} \sum_{a b c} \varepsilon_{\mu \nu \rho \sigma} c^{a}(n) F_{\mu \nu}^{b}(n) F_{\rho \sigma}^{c}(n+\widehat{\mu}+\widehat{\nu})+\delta_{B} \mathcal{B}[A],
$$

where the functional $\mathcal{B}$ depends smoothly and locally on the gauge potential $A_{\mu}^{a}$.

\footnotetext{
** Since the fermion determinant Det $M[A]$ is gauge invariant, one can then regard it as a functional of the link variable Det $M[U]$ for the case I (this is trivially the case for the case II).
} 
This is a natural generalization of Lüscher's result (1.1) to multi- $U(1)$ cases. Thus, under the prerequisites of the theorem, the anomaly cancellation in the corresponding continuum theory $\sum_{R} e_{R}^{a} e_{R}^{b} e_{R}^{c}-\sum_{L} e_{L}^{a} e_{L}^{b} e_{L}^{c}=0$ guarantees the gauge invariance of the effective action, after subtracting the local counterterm $\mathcal{B}$.

For nonabelian gauge theories, we will show the following statement.

Theorem (Nonabelian theory). Let $\mathcal{A}[c, A]$ be the gauge anomaly defined on a 4dimensional infinite hypercubic lattice. Suppose that (I) $\mathcal{A}$ depends smoothly and locally on the gauge potential $A_{\mu}$ and on the ghost field $c$, (II) $\mathcal{A}$ reproduces for smooth field configurations the gauge anomaly in the continuum theory (2.28) in the classical continuum limit, and (III) $U(1)$ gauge anomalies in $\mathcal{A}$ have the topological property

$$
\delta \mathcal{A}=\left(\text { terms proportional to } c^{(\alpha)}\right), \quad \text { for } \quad c^{U(1)_{\beta}}(n) \rightarrow \text { const. }
$$

where $\delta$ is an arbitrary local variation of the gauge potential. Then if the anomaly in the corresponding continuum theory cancels, $\operatorname{tr}_{R-L} T^{a}\left\{T^{b}, T^{c}\right\}=0$ etc., $\mathcal{A}$ is always BRS trivial, i.e., $\mathcal{A}=\delta_{B} \mathcal{B}[A]$, where the functional $\mathcal{B}$ depends smoothly and locally on the gauge potential $A_{\mu}$. This statement holds to all orders in powers of the gauge potential $A_{\mu}$. The explicit form of the nontrivial anomaly $\mathcal{A} \neq \delta_{B} \mathcal{B}$ is given in eq. (7.50).

Therefore, to all orders in powers of the gauge potential, the anomaly cancellation in the continuum theory guarantees that of the lattice theory. This seems remarkable but is not entirely unexpected. Let us recall the expression in the classical continuum limit (2.28). In fact the expression holds to all orders in powers of the lattice spacing $a$ in the classical continuum limit $a \rightarrow 0$ (we assume that the Lorentz covariance is restored in this limit). In the classical continuum limit, each coefficient of the expansion with respect to $a$ is a local functional of the gauge potential and the ghost field. Then the uniqueness theorem of nontrivial anomalies in the continuum theory $[16,17]$ can be invoked and one concludes that the anomaly (2.28) is the unique possibility (up to contributions of local counterterms). Therefore the anomaly cancellation $\operatorname{tr}_{R-L} T^{a}\left\{T^{b}, T^{c}\right\}=0$ guarantees that $\mathcal{A}=\delta_{B} \mathcal{B}$ to all orders in powers of the lattice spacing $a$ in the classical continuum limit $a \rightarrow 0$. Of course the expansion with respect to $a$ is (presumably at most) asymptotic and this does not prove the anomaly cancellation for $a \neq 0$. Nevertheless, this argument makes the content of the 
above theorem quite plausible. Finally, we emphasize that the above theorems themselves do not assume the Ginsparg-Wilson relation such that they are applicable to any formulation if the prerequisites of the theorems are fulfilled.

\section{Preliminaries in the abelian theory}

\subsection{Noncommutative Differential CAlculus}

To determine general nontrivial local solutions to the consistency condition (1.3), we need cohomological information, as in the continuum theory [16,17]. To discuss $d$-cohomology on an infinite lattice, the technique of noncommutative differential calculus [28-31] is very useful, because it makes the standard Leibniz rule of the exterior derivative valid even on the lattice. In fact, this technique was applied successfully $[32,33]$ to an algebraic proof of the higher dimensional extension of Lüscher's theorem of ref. [19] (which is basically equivalent to eq. (1.1)). Here we recapitulate its basic setup.

The bases of the 1-form on D-dimensional infinite hypercubic lattice are defined as objects which satisfy the Grassmann algebra

$$
d x_{1}, d x_{2}, \cdots, d x_{D}, \quad d x_{\mu} d x_{\nu}=-d x_{\nu} d x_{\mu}
$$

A generic $p$-form is defined by

$$
f(n)=\frac{1}{p !} f_{\mu_{1} \cdots \mu_{p}}(n) d x_{\mu_{1}} \cdots d x_{\mu_{p}}
$$

where the summation over repeated indices is understood. The exterior derivative is then defined by the forward difference operator as

$$
d f(n)=\frac{1}{p !} \Delta_{\mu} f_{\mu_{1} \cdots \mu_{p}}(n) d x_{\mu} d x_{\mu_{1}} \cdots d x_{\mu_{p}} .
$$

The nilpotency of the exterior derivative $d^{2}=0$ follows from this definition. The essence of the noncommutative differential calculus on infinite lattice is

$$
d x_{\mu} f(n)=f(n+\widehat{\mu}) d x_{\mu}
$$

where $f(n)$ is a 0 -form (i.e., a function). That is, a function on the lattice and the basis of a 1-form do not simply commute. The argument of the function is shifted along $\mu$-direction by 
one unit when commuting these two objects. The remarkable fact, which follows from the noncommutativity (4.4), is that the standard Leibniz rule of the exterior derivative $d$ holds. With eqs. (4.3) and (4.4), one can easily confirm that

$$
d[f(n) g(n)]=d f(n) g(n)+(-1)^{p} f(n) d g(n),
$$

for forms $f(n)$ and $g(n)$ where $f(n)$ is a $p$-form. The validity of this Leibniz rule is quite helpful for following analyses.

We also introduce the abelian gauge potential 1-form and the abelian field strength 2-form by

$$
A^{a}(n)=A_{\mu}^{a}(n) d x_{\mu}, \quad F^{a}(n)=\frac{1}{2} F_{\mu \nu}^{a}(n) d x_{\mu} d x_{\nu}=d A^{a}(n) .
$$

Note that the Bianchi identity takes the form $d F^{a}(n)=0$. We will never use the symbol $F^{a}$ or $F$ for nonabelian field strength 2-form.

\subsection{Abelian BRS transformation}

Using eqs. (1.2) and (2.6), the BRS transformation for the gauge potential and for the ghost field in the abelian theory is given by

$$
\delta_{B} A_{\mu}^{a}(n)=\Delta_{\mu} c^{a}(n), \quad \delta_{B} c^{a}(n)=0 .
$$

The BRS transformation is nilpotent $\delta_{B}^{2}=0$ and the abelian field strength is BRS invari-

ant $\delta_{B} F_{\mu \nu}^{a}(n)=0$. We also introduce the Grassmann coordinate $\theta$ [58-60] and define the BRS exterior derivative by

$$
s=\delta_{B} d \theta
$$

The usual 1-form $d x_{\mu}$ and the BRS 1-form $d \theta$ anticommute with each other $d x_{\mu} d \theta=-d \theta d x_{\mu}$ and the BRS 1-form $d \theta$ commutes with itself $d \theta d \theta \neq 0$. Therefore, for a Grassmann-even 
(-odd) $p$-form $f(n)$, we have

$$
d \theta f(n)= \pm(-1)^{p} f(n) d \theta .
$$

We also have

$$
s^{2}=\{s, d\}=0,
$$

where the first relation follows from $\delta_{B}^{2}=0$. Finally, we introduce the ghost 1 -form by

$$
C^{a}(n)=c^{a}(n) d \theta .
$$

In terms of these forms, the BRS transformation in the abelian theory (4.7) is expressed as

$$
s A^{a}(n)=-d C^{a}(n), \quad s C^{a}(n)=0, \quad s F^{a}(n)=0 .
$$

The noncommutative rule (4.4) will always be assumed in expressions written in terms of differential forms.

\section{Basic lemmas in the abelian theory}

Using the tools introduced in the preceding section, we establish in this section several lemmas which provide cohomological information. The algebraic Poincaré lemma specifies the $d$-cohomology on local functions of the gauge potential and the ghost field. We use the Poincaré lemma on an infinite lattice [19], which might be regarded as a triviality of the de Rham cohomology, to prove this lemma. We next determine the BRS cohomology in the abelian theory $G=U(1)^{N}$. Finally the covariant Poincaré lemma tells the $d$-cohomology on $s$-invariant functions. In the terminology of ref. [17], these three lemmas correspond to $\mathrm{H}(d), \mathrm{H}(\delta)$ and $\mathrm{H}(\mathrm{H}(\delta), d)$, respectively.

\subsection{Algebraic Poincaré lemma}

The algebraic Poincaré lemma ${ }^{\star}$ asserts that a $d$-closed form-valued local function on a $D$ dimensional infinite lattice is always $d$-exact up to a constant form; $D$-forms are exceptional because any $D$-form is $d$-closed. Moreover, the lemma asserts that the locality is preserved between the original form and its "ancestor."

\footnotetext{
$\star$ The present algebraic Poincaré lemma is somewhat different from that of ref. [32]. Practically, the present form is more convenient.
} 
Algebraic Poincaré lemma. Let $\eta$ be a $p$-form on a $D$-dimensional infinite lattice that depends smoothly and locally on the gauge potential $A_{\mu}^{a}$ and on the ghost field $c^{a}$. Then

$$
d \eta(n)=0 \Leftrightarrow \eta(n)=d \chi(n)+\mathcal{L}(n) d^{D} x+B
$$

where $B$ is a constant $p$-form and the $(p-1)$-form $\chi$ and the function $\mathcal{L}$ depend smoothly and locally on the gauge potential and on the ghost field. The function $\mathcal{L}$ satisfies

$$
\sum_{n} \delta \mathcal{L}(n) \neq 0
$$

for a certain local variation $\delta$ of the gauge potential and the ghost field.

Note. The term $\mathcal{L} d^{D} x$ in eq. (5.1) represents a non-topological part in the $D$-form $\eta$. In other words, a $D$-form $\eta_{\text {top. }}$ that is topological, $\sum_{n} \delta \eta_{\text {top. }}(n)=0$ for an arbitrary local variation, is always $d$-exact up to a constant form.

Proof. We define $\eta_{t}$ by rescaling fields as $A_{\mu}^{a} \rightarrow t A_{\mu}^{a}$ and $c^{a} \rightarrow t c^{a}$. Then, since $\eta$ depends smoothly on $A_{\mu}^{a}$ and on $c^{a}$,

$$
\begin{aligned}
\eta(n) & =\eta(n)_{t=0}+\int_{0}^{1} d t \frac{\partial \eta(n)_{t}}{\partial t} \\
& =\eta(n)_{t=0}+\sum_{n^{\prime}}\left[A_{\mu}^{a}\left(n^{\prime}\right) \theta_{\mu}^{a}\left(n^{\prime}, n\right)+c^{a}\left(n^{\prime}\right) \kappa^{a}\left(n^{\prime}, n\right)\right]
\end{aligned}
$$

where

$$
\theta_{\mu}^{a}\left(n^{\prime}, n\right)=\int_{0}^{1} d t \frac{\partial \eta(n)_{t}}{\partial t A_{\mu}^{a}\left(n^{\prime}\right)}, \quad \kappa^{a}\left(n^{\prime}, n\right)=\int_{0}^{1} d t \frac{\partial \eta(n)_{t}}{\partial t c^{a}\left(n^{\prime}\right)}
$$

Eq. (5.4) implies

$$
d \theta_{\mu}^{a}\left(n^{\prime}, n\right)=d \kappa^{a}\left(n^{\prime}, n\right)=0
$$

because $d \eta=0$ for arbitrary configurations. Moreover, since $\eta$ depends locally on $A_{\mu}^{a}$ and on $c^{a}, \theta_{\mu}^{a}\left(n^{\prime}, n\right)$ and $\kappa^{a}\left(n^{\prime}, n\right)$ decay exponentially as $\left|n-n^{\prime}\right| \rightarrow \infty$. This allows us to apply 
Lüscher's Poincaré lemma [19] for $p<D$ to eq. (5.5) which asserts that there exist forms $\Theta_{\mu}^{a}$ and $K^{a}$ such that

$$
\theta_{\mu}^{a}\left(n^{\prime}, n\right)=d \Theta_{\mu}^{a}\left(n^{\prime}, n\right), \quad \kappa^{a}\left(n^{\prime}, n\right)=d K^{a}\left(n^{\prime}, n\right) .
$$

These forms $\Theta_{\mu}^{a}\left(n^{\prime}, n\right)$ and $K^{a}\left(n^{\prime}, n\right)$ also decay exponentially as $\left|n-n^{\prime}\right| \rightarrow \infty$ [19]. Substituting this into eq. (5.3), we have $\eta=d \chi+B$, where $B=\eta_{t=0}$, and

$$
\chi(n)=\sum_{n^{\prime}}\left[A_{\mu}^{a}\left(n^{\prime}\right) \Theta_{\mu}^{a}\left(n^{\prime}, n\right)+c^{a}\left(n^{\prime}\right) K^{a}\left(n^{\prime}, n\right)\right] .
$$

From the locality property of $\Theta_{\mu}^{a}\left(n^{\prime}, n\right)$ and of $K^{a}\left(n^{\prime}, n\right)$ [19], one can easily see [32] that $\chi(n)$ is a local field. Also the smoothness is preserved in the construction (5.7). In this way, the lemma (5.1) is established for $p<D$.

For $p=D, d \eta=0$ is a trivial statement and thus we decompose $\eta$ as

$$
\eta=\eta_{\text {top. }}+\mathcal{L} d^{D} x
$$

where $\sum_{n} \delta \eta_{\text {top. }}(n)=0$ for an arbitrary local variation. Then $\theta_{\mu}^{a}$ and $\kappa^{a}$ in eq. (5.4) defined from $\eta_{\text {top. }}$ satisfy

$$
\sum_{n} \theta_{\mu}^{a}\left(n^{\prime}, n\right)=\sum_{n} \kappa^{a}\left(n^{\prime}, n\right)=0 .
$$

Then Lüscher's Poincaré lemma for $p=D$ [19] asserts that there exist $\Theta_{\mu}^{a}$ and $K^{a}$ which satisfy eq. (5.6). The rest is the same as for $p<D$ and we have $\eta_{\text {top. }}=d \chi+B$.

\subsection{Abelian BRS COHOMOLOGy}

Abelian BRS cohomology. Let $X$ be a form on infinite hypercubic lattice that depends smoothly and locally on the gauge potential and on the ghost field. Then,

$$
s X(n)=0 \Leftrightarrow X(n)=C^{a_{1}}(n) \cdots C^{a_{g}}(n) X_{0}^{\left[a_{1} \cdots a_{g}\right]}\left[\left\{F_{i}\right\} ; n\right]+s Y(n),
$$

where the form $X_{0}^{\left[a_{1} \cdots a_{g}\right]}(n)$ depends smoothly and locally only on the abelian field strength $F_{\mu \nu}^{a}$. The form $Y(n)$ depends smoothly and locally on the gauge potential and 
on the ghost field. In particular, differences of the ghost field can appear only in the BRS trivial part $s Y$.

Note. The form $X_{0}^{\left[a_{1} \cdots a_{g}\right]}$ is totally antisymmetric on the upper indices because ghost 1-

forms $C^{a}$ simply anticommute with each other. $X_{0}^{\left[a_{1} \cdots a_{g}\right]}(n)$ depends only on the field strength $F_{\mu \nu}^{a}(n)$ and its differences, such as $\Delta_{\mu} F_{\nu \rho}^{a}(n), \Delta_{\mu}^{*} F_{\nu \rho}^{a}(n), \Delta_{\mu} \Delta_{\nu} F_{\rho \sigma}^{a}(n)$ and so on; obviously $X_{0}^{\left[a_{1} \cdots a_{g}\right]}$ is gauge invariant. In what follows, we denote as $X_{0}^{\left[a_{1} \cdots a_{g}\right]}\left[\left\{F_{i}\right\}\right]$ to indicate this particular dependence on the field strength, including smoothness and locality of the dependence.

Proof. The proof of the abelian BRS cohomology for a single $U(1)$ case [32] can be repeated by simply supplementing the gauge potential $A_{\mu}$ and the ghost field $c$ by another index $a$. Thus we do not reproduce it here to save the space.

\subsection{Covariant Poincaré lemma}

As in the continuum theory [16], the following covariant Poincaré lemma is crucial to determine general nontrivial local solutions to the consistency condition. This lemma for a single $U(1)$ case $G=U(1)$ was given in ref. [32]. It turns out that, however, its extension to multi- $U(1)$ cases is not trivial, due to the reason which will be explained after the proof. In fact, we have at present only the following cumbersome proof that works only for 4- or lower dimensional lattice.

Covariant Poincaré lemma. On a 4-dimensional infinite hypercubic lattice, if the $p$ form $\alpha_{p}\left[\left\{F_{i}\right\}\right]$ is $d$-closed for $p<4$, or if $\alpha_{4}\left[\left\{F_{i}\right\}\right]=d \chi_{3}+B_{4}$ where $B_{4}$ is a constant 4-form, then $\alpha_{p}$ is of the structure

$$
\alpha_{p}\left[\left\{F_{i}\right\} ; n\right]=d \alpha_{p-1}\left[\left\{F_{i}\right\} ; n\right]+B_{p}+F^{a}(n) B_{p-2}^{a}+F^{a}(n) F^{b}(n) B_{p-4}^{(a b)},
$$

where $F^{a}$ is the field strength 2-form and $B$ 's are constant forms.

Note. Here all expressions are written in terms of the noncommutative differential calculus. Proof. We prove the lemma step by step from 0 -form $p=0$ until 4 -form $p=4$. 
For $p=0$. The lemma trivially holds by the algebraic Poincaré lemma (5.1). Namely, the $d$-closed 0 -form $\alpha_{0}$ must be a constant $\alpha_{0}=B_{0}$.

For $p=1$. By the algebraic Poincaré lemma, the $d$-closed 1-form $\alpha_{1}$ is $d$-exact up to a constant 1 -form. Also $\alpha_{1}$ is $s$-closed because it is a function of the field strength. Namely,

$$
\alpha_{1}=d \chi_{0}^{0}+B_{1}, \quad s \alpha_{1}=0
$$

Since these equations imply $s \alpha_{1}=s d \chi_{0}^{0}=-d s \chi_{0}^{0}=0$, the algebraic Poincaré lemma asserts that

$$
s \chi_{0}^{0}=0
$$

where we have used the fact that the right hand side cannot be a constant. The solution to this equation is given by the abelian BRS cohomology (5.10) for the $g=0$ case:

$$
\chi_{0}^{0}=\omega_{0}\left[\left\{F_{i}\right\}\right]
$$

and thus eq. (5.12) shows that the lemma holds for $p=1$ :

$$
\alpha_{1}=d \omega_{0}\left[\left\{F_{i}\right\}\right]+B_{1}
$$

$\underline{\text { For } p=2}$. In this case, from the algebraic Poincaré lemma, we have

$$
\alpha_{2}=d \chi_{1}^{0}+B_{1}, \quad s \alpha_{2}=0
$$

and, in a similar way as the $p=1$ case, these lead to the following descent equations

$$
s \chi_{1}^{0}=d \chi_{0}^{1}, \quad s \chi_{0}^{1}=0
$$

The general solution to the last equation is given by the abelian BRS cohomology

$$
\chi_{0}^{1}=C^{a} \omega_{0}^{a}\left[\left\{F_{i}\right\}\right]+s \beta_{0} .
$$

We may, however, absorb $\beta_{0}$ in redefinition of $\chi_{0}^{1}$ and $\chi_{1}^{0}$,

$$
\chi_{0}^{1} \rightarrow \chi_{0}^{1}+s \beta_{0}, \quad \chi_{1}^{0} \rightarrow \chi_{1}^{0}-d \beta_{0},
$$

without changing $\alpha_{2}$. We can therefore take $\chi_{0}^{1}=C^{a} \omega_{0}^{a}$. Then the first equation in eq. (5.17) 
reads

$$
\begin{aligned}
s \chi_{1}^{0} & =d C^{a} \omega_{0}^{a}-C^{a} d \omega_{0}^{a} \\
& =-s\left(A^{a} \omega_{0}^{a}\right)-C^{a} d \omega_{0}^{a} .
\end{aligned}
$$

Now consider a special configuration of the ghost field $c^{a}(n) \rightarrow c^{a}=$ const. Then the consistency of eq. (5.20) requires

$$
d \omega_{0}^{a}=0, \quad s\left(\chi_{1}^{0}+A^{a} \omega_{0}^{a}\right)=0,
$$

because $s$ (something) is proportional to differences of the ghost fields such as $c^{a}(n+\widehat{\mu})-$ $c^{a}(n)$. Note that $\omega_{0}^{a}$ does not depend on the ghost field. The solution to the first equation of eq. (5.21) is given by the present lemma for $p=0$, which we have shown above:

$$
\omega_{0}^{a}=B_{0}^{a} \quad \text { (const.) }
$$

and then the second relation of eq. (5.21) implies

$$
\chi_{1}^{0}=-A^{a} B_{0}^{a}+\omega_{1}\left[\left\{F_{i}\right\}\right]
$$

by the BRS cohomology. Going back to the original relation (5.16), we have

$$
\alpha_{2}=-F^{a} B_{0}^{a}+d \omega_{1}\left[\left\{F_{i}\right\}\right]+B_{2}
$$

because $d A^{a}=F^{a}$. This proves the lemma for $p=2$.

$\underline{\text { For } p=3}$. In this case, the counterparts of eqs. (5.16) and (5.17) are

$$
\alpha_{3}=d \chi_{2}^{0}+B_{3}, \quad s \alpha_{3}=0,
$$

and

$$
s \chi_{2}^{0}=d \chi_{1}^{1}, \quad s \chi_{1}^{1}=d \chi_{0}^{2}, \quad s \chi_{0}^{2}=0 .
$$

The solution to the last equation is (we have absorbed the BRS trivial part as eq. (5.19))

$$
\chi_{0}^{2}=C^{a} C^{b} \omega_{0}^{[a b]}\left[\left\{F_{i}\right\}\right]
$$

where $\omega_{0}^{[a b]}$ is antisymmetric under $a \leftrightarrow b$. At this stage, it is quite convenient to introduce 
the symmetrization symbol defined by

$$
\operatorname{sym}\left(X_{1} X_{2} \cdots X_{N}\right)=\sum_{\sigma} \frac{1}{N !} \epsilon_{\sigma} X_{\sigma(1)} X_{\sigma(2)} \cdots X_{\sigma(N)}
$$

where the summation is taken over all permutations $\sigma$. The sign factor $\epsilon_{\sigma}$ is defined as the signature arising when the product $X_{1} \cdots X_{N}$ is converted to the order $X_{\sigma(1)} X_{\sigma(2)} \cdots X_{\sigma(N)}$ by regarding all $X_{i}$ 's as ordinary forms (i.e., the form basis $d x_{\mu}$ simply commutes with functions). Using the symmetrization symbol, eq. (5.27) is trivially written as

$$
\chi_{0}^{2}=\operatorname{sym}\left(C^{a} C^{b}\right) \omega_{0}^{[a b]}
$$

and then the second relation of eq. (5.26) reads

$$
\begin{aligned}
s \chi_{1}^{1} & =2 \operatorname{sym}\left(d C^{a} C^{b}\right) \omega_{0}^{[a b]}+\operatorname{sym}\left(C^{a} C^{b}\right) d \omega_{0}^{[a b]} \\
& =s\left[-2 \operatorname{sym}\left(A^{a} C^{b}\right) \omega_{0}^{[a b]}\right]+\operatorname{sym}\left(C^{a} C^{b}\right) d \omega_{0}^{[a b]} .
\end{aligned}
$$

Let us now consider the special configuration $c^{a}(n) \rightarrow$ const. As for eq. (5.20), the consistency of eq. (5.30) requires

$$
d \omega_{0}^{[a b]}=0, \quad s\left[\chi_{1}^{1}+2 \operatorname{sym}\left(A^{a} C^{b}\right) \omega_{0}^{[a b]}\right]=0
$$

The general solution to the first equation is $\omega_{0}^{[a b]}=B_{0}^{[a b]}$ and then the second equation implies (by the BRS cohomology) $\chi_{1}^{1}=-2 \operatorname{sym}\left(A^{a} C^{b}\right) B_{0}^{[a b]}+C^{a} \omega_{1}^{a}\left[\left\{F_{i}\right\}\right]$. Substituting these into the first relation of eq. (5.26), we have

$$
s \chi_{2}^{0}=s\left[\operatorname{sym}\left(A^{a} A^{b}\right) B_{0}^{[a b]}-A^{a} \omega_{1}^{a}\right]-2 \operatorname{sym}\left(F^{a} C^{b}\right) B_{0}^{[a b]}-C^{a} d \omega_{1}^{a} .
$$

We again consider the configuration $c^{a}(n) \rightarrow$ const. Then eq. (5.32) requires

$$
2 F^{a} B_{0}^{[a b]}+d \omega_{1}^{b}=0, \quad s\left[\chi_{2}^{0}-\operatorname{sym}\left(A^{a} A^{b}\right) B_{0}^{[a b]}+A^{a} \omega_{1}^{a}\right]=0 .
$$

In deriving the first relation, we have noted the fact that the constant ghost form $C^{b}$ and the 2-form $F^{a}$ simply commute, and thus $C^{b}$ can be factored out from the equation. Next 
we consider a configuration $F_{\mu \nu}^{a}(n) \rightarrow$ const. Since $\omega_{1}^{a}$ depends only on the field strength, we see that the constant $B_{0}^{[a b]}$ must vanish for the consistency of eq. $(5.33)^{\star}$ and thus

$$
d \omega_{1}^{a}=0 \Rightarrow \omega_{1}^{a}=d \omega_{0}^{a}\left[\left\{F_{i}\right\}\right]+B_{1}^{a},
$$

by the present lemma for $p=1$. Substituting this into the second relation of eq. (5.33) and using the first equation (5.25), we have

$$
\begin{aligned}
\alpha_{3} & =-F^{a} d \omega_{0}^{a}-F^{a} B_{1}^{a}+d \omega_{2}+B_{3} \\
& =d\left(-F^{a} \omega_{0}^{a}+\omega_{2}\right)-F^{a} B_{1}^{a}+B_{3},
\end{aligned}
$$

where we have used the Bianchi identity $d F^{a}=0$. This shows the lemma for $p=3$.

For $p=4$. Similarly as the above cases, we have

$$
\alpha_{4}=d \chi_{3}^{0}+B_{4}, \quad s \alpha_{4}=0
$$

and

$$
s \chi_{3}^{0}=d \chi_{2}^{1}, \quad s \chi_{2}^{1}=d \chi_{1}^{2}, \quad s \chi_{1}^{2}=d \chi_{0}^{3}, \quad s \chi_{0}^{3}=0 .
$$

The solution to the last equation is given by $\chi_{0}^{3}=\operatorname{sym}\left(C^{a} C^{b} C^{c}\right) \omega_{0}^{[a b c]}\left[\left\{F_{i}\right\}\right]$ and then the third relation of eq. (5.37) reads

$$
s \chi_{1}^{2}=s\left[-3 \operatorname{sym}\left(A^{a} C^{b} C^{c}\right) \omega_{0}^{[a b c]}\right]-\operatorname{sym}\left(C^{a} C^{b} C^{c}\right) d \omega_{0}^{[a b c]} .
$$

The consistency for $c^{a}(n) \rightarrow$ const. requires $\omega_{0}^{[a b c]}=B_{0}^{[a b c]}$ (const.) and thus

$$
\chi_{1}^{2}=-3 \operatorname{sym}\left(A^{a} C^{b} C^{c}\right) B_{0}^{[a b c]}+\operatorname{sym}\left(C^{a} C^{b}\right) \omega_{1}^{[a b]}\left[\left\{F_{i}\right\}\right],
$$

and the second equation of eq. (5.37) becomes

$$
\begin{aligned}
s \chi_{2}^{1}=s[ & \left.-3 \operatorname{sym}\left(A^{a} A^{b} C^{c}\right) B_{0}^{[a b c]}-2 \operatorname{sym}\left(A^{a} C^{b}\right) \omega_{1}^{[a b]}\right] \\
& -3 \operatorname{sym}\left(F^{a} C^{b} C^{c}\right) B_{0}^{[a b c]}+\operatorname{sym}\left(C^{a} C^{b}\right) d \omega_{1}^{[a b]} .
\end{aligned}
$$

Setting $c^{a}(n) \rightarrow$ const. in this equation and then setting $F_{\mu \nu}^{a}(n) \rightarrow$ const., we see that $B_{0}^{[a b c]}=0$ and $d \omega_{1}^{[a b]}=0$. The present lemma for $p=1$ then asserts that $\omega_{1}^{[a b]}=d \omega_{0}^{[a b]}\left[\left\{F_{i}\right\}\right]+$

* Later, we apply the covariant Poincaré lemma to the case II above, by regarding components of the nonabelian gauge potential $A_{\mu}(n)=\sum_{a} A_{\mu}^{a}(n) T^{a}$ as if they were the abelian gauge potential. In this case, it is impossible to take $F_{\mu \nu}^{a}(n)=$ const. while keeping the range of $A_{\mu}^{a}(n)$ as eq. (2.9). However, it is possible to take $F_{\mu \nu}^{a}(n)=$ const. $=O(1 / R)$ inside of a block of size $R$. The term $d \omega_{1}^{b}$ then behaves as $\sim \exp (-\alpha R)$ because the dependence of $\omega_{1}^{b}$ is local. Since the first relation of eq. (5.33) holds for arbitrary $R$, this implies that each term has to vanish separately. 
$B_{1}^{[a b]}$. The general structure of $\chi_{2}^{1}$ is therefore given by

$$
\chi_{2}^{1}=-2 \operatorname{sym}\left(A^{a} C^{b}\right)\left(d \omega_{0}^{[a b]}+B_{1}^{[a b]}\right)+C^{a} \omega_{2}^{a}\left[\left\{F_{i}\right\}\right] .
$$

Substituting this into the first relation of eq. (5.37), we have

$$
\begin{aligned}
s \chi_{3}^{0}=s[ & \left.\operatorname{sym}\left(A^{a} A^{b}\right)\left(d \omega_{0}^{[a b]}+B_{1}^{[a b]}\right)-A^{a} \omega_{2}^{a}\right] \\
& -2 \operatorname{sym}\left(F^{a} C^{b}\right)\left(d \omega_{0}^{[a b]}+B_{1}^{[a b]}\right)-C^{a} d \omega_{2}^{a} .
\end{aligned}
$$

The consistency for $c^{a}(n) \rightarrow$ const. requires

$$
2 F^{a}\left(d \omega_{0}^{[a b]}+B_{1}^{[a b]}\right)+d \omega_{2}^{b}=0,
$$

and the consistency for $F_{\mu \nu}^{a}(n) \rightarrow$ const.,

$$
B_{1}^{[a b]}=0, \quad 2 F^{a} d \omega_{0}^{[a b]}+d \omega_{2}^{b}=0 .
$$

The last equation can be written as $d\left(\omega_{2}^{a}-2 F^{b} \omega_{0}^{[a b]}\right)=0$, and then the present lemma for $p=2$ asserts that

$$
\omega_{2}^{a}=2 F^{b} \omega_{0}^{[a b]}+d \omega_{1}^{a}\left[\left\{F_{i}\right\}\right]+B_{2}^{a}+F^{b} B_{0}^{a b}
$$

Note that $B_{0}^{a b}$ is not necessarily symmetric under $a \leftrightarrow b$ at this stage. From this it is not difficult to see that eq. (5.42) yields

$$
\begin{gathered}
s \chi_{3}^{0}=s d\left[\operatorname{sym}\left(A^{a} A^{b}\right) \omega_{0}^{[a b]}\right]+s\left[-A^{a}\left(d \omega_{1}^{a}+B_{2}^{a}+F^{b} B_{0}^{a b}\right)\right] \\
+d\left\{2\left[\operatorname{sym}\left(F^{a} C^{b}\right)+C^{a} F^{b}\right] \omega_{0}^{[a b]}\right\} .
\end{gathered}
$$

We have now arrived at the final stage which requires special consideration. In eq. (5.46), the last term on the right hand side is not manifestly $s$-exact. So define

$$
\varphi_{2}^{1}=2\left[\operatorname{sym}\left(F^{a} C^{b}\right)+C^{a} F^{b}\right] \omega_{0}^{[a b]}=\left(C^{a} F^{b}-F^{b} C^{a}\right) \omega_{0}^{[a b]} .
$$

In the context of ordinary differential calculus, $\varphi_{2}^{1}$ identically vanishes because $C^{a}$ and $F^{b}$ commute with each other. However we cannot simply throw away $\varphi_{2}^{1}$ in the context of 
noncommutative differential calculus. We first note $s \varphi_{2}^{1}=0$. Also, when $c^{a}(n) \rightarrow$ const., $C^{a}$ and $F^{b}$ commute and $\varphi_{2}^{1}=0$ as noted above. Therefore $\varphi_{2}^{1} \propto \Delta_{\mu} c^{a}$. These facts combined with the BRS cohomology (5.10) show that $\varphi_{2}^{1}$ is $s$-trivial, $\varphi_{2}^{1}=s Y_{2}$ (actually, otherwise eq. (5.46) becomes inconsistent). In fact, by noting the noncommutative rule (4.4), one finds

$$
\varphi_{2}^{1}=-\left[\Delta_{\mu} c^{a}(n)+\Delta_{\nu} c^{a}(n)+\Delta_{\mu} \Delta_{\nu} c^{a}(n)\right] d \theta \frac{1}{2} F_{\mu \nu}^{b}(n) d x_{\mu} d x_{\nu} \omega_{0}^{[a b]}=s Y_{2},
$$

where

$$
Y_{2}=-\left[A_{\mu}^{a}(n)+A_{\nu}^{a}(n)+\Delta_{\nu} A_{\mu}^{a}(n)\right] \frac{1}{2} F_{\mu \nu}^{b}(n) d x_{\mu} d x_{\nu} \omega_{0}^{[a b]} .
$$

Therefore eq. (5.46) gives

$$
\chi_{3}^{0}=-A^{a}\left(d \omega_{1}^{a}+B_{2}^{a}+F^{b} B_{0}^{a b}\right)+\omega_{3}\left[\left\{F_{i}\right\}\right]+d\left[\operatorname{sym}\left(A^{a} A^{b}\right) \omega_{0}^{[a b]}-Y_{2}\right],
$$

and from the first equation (5.36), we have

$$
\alpha_{4}=d\left(-F^{a} \omega_{1}^{a}+\omega_{3}\right)-F^{a} B_{2}^{a}-F^{a} F^{b} B_{0}^{a b}+B_{4} .
$$

Finally, we show that the term which is proportional to the antisymmetric part of $B_{0}^{a b}$ under $a \leftrightarrow b$, and which again vanishes in ordinary differential calculus, can be expressed as $d \varphi_{3}\left(\left\{F_{i}\right\}\right)$. First note that $F^{a} F^{b} B_{0}^{[a b]}=d \varphi_{3}$ where

$$
\varphi_{3}=\frac{1}{2}\left(A^{a} F^{b}-F^{b} A^{a}\right) B_{0}^{[a b]}-\frac{1}{2} d Y_{2},
$$

and $Y_{2}$ in the second term is defined by $\omega_{0}^{[a b]} \rightarrow B_{0}^{[a b]}$ in eq. (5.49). Of course, the last term $-d Y_{2} / 2$ does dot contribute to $F^{a} F^{b} B_{0}^{[a b]}$, but it makes $\varphi_{3}$ gauge invariant. In fact,

$$
s \varphi_{3}=-\frac{1}{2}\left(d C^{a} F^{b}-F^{b} d C^{a}\right) B_{0}^{[a b]}+\frac{1}{2} d s Y_{2}=0,
$$

where use of eqs. (5.48) and (5.47) has been made. More explicitly, after some calculation with use of the Bianchi identity, we have

$$
\begin{aligned}
\varphi_{3}(n)=\frac{1}{8}[ & F_{\alpha \beta}^{a}(n) F_{\beta \gamma}^{b}(n)+F_{\alpha \beta}^{a}(n+\widehat{\gamma}) F_{\beta \gamma}^{b}(n) \\
& \left.\quad+F_{\alpha \beta}^{a}(n) F_{\beta \gamma}^{b}(n+\widehat{\alpha})+F_{\alpha \beta}^{a}(n+\widehat{\gamma}) F_{\beta \gamma}^{b}(n+\widehat{\alpha})\right] d x_{\alpha} d x_{\beta} d x_{\gamma} B_{0}^{[a b]} .
\end{aligned}
$$

This establishes the lemma for $p=4$. 
If one repeats the above argument for $p=5$ (assuming that the dimension of the lattice is greater than 4), the treatment becomes much more involved due to the noncommutativity of forms. Because of this, we could not find an iterative formula for $\alpha_{p}$ with general $p$, unlike the treatment in the continuum theory [16]. This fact suggests that our noncommutative differential calculus is not powerful enough and there must exist another hidden algebraic structure. This is an interesting problem although we do not investigate it here. Of course, the proof in this subsection is sufficient for applications on 4-dimensional lattices.

\subsection{TOPOLOGICAL FIELDS IN THE ABELIAN THEORY}

Once the above three lemmas are established, it is straightforward to show the following theorem which generalizes the theorem of ref. [19] to multi- $U(1)$ cases.

Theorem. Let $q(n)$ be a gauge invariant field on a 4-dimensional infinite hypercubic lattice

that depends smoothly and locally on the abelian gauge potential $A_{\mu}^{a}$. Suppose that $q(n)$ is topological, namely

$$
\sum_{n} \delta q(n)=0
$$

for an arbitrary local variation of the gauge potential. Then $q(n)$ is of the form

$$
q(n)=\alpha+\beta_{\mu \nu}^{a} F_{\mu \nu}^{a}(n)+\gamma^{(a b)} \varepsilon_{\mu \nu \rho \sigma} F_{\mu \nu}^{a}(n) F_{\rho \sigma}^{b}(n+\widehat{\mu}+\widehat{\nu})+\Delta_{\mu}^{*} k_{\mu}(n),
$$

where the current $k_{\mu}(n)$ depends smoothly and locally only on the field strength and thus is gauge invariant.

Proof. We multiply the volume form $d^{4} x$ to $q(n)$ and define the 4 -form $Q_{4}=q d^{4} x$. $Q_{4}$ is gauge invariant $s Q_{4}=0$ and thus, from the BRS cohomology (5.10), $Q_{4}=Q_{4}\left(\left\{F_{i}\right\}\right)$. From the algebraic Poincaré lemma (5.1), on the other hand, $Q_{4}=d \chi_{3}+B_{4}$ because the 4-form $Q_{4}$ is topological $\sum_{n} \delta Q_{4}(n)=0$ from the assumption (5.55). From these, we can apply the covariant Poincaré lemma (5.11) to $Q_{4}$ which yields

$$
q(n) d^{4} x=Q_{4}(n)=B_{4}+F^{a}(n) B_{2}^{a}+F^{a}(n) F^{b}(n) B_{0}^{(a b)}+d \alpha_{3}(n) .
$$

Finally, we factor out the volume form $d^{4} x$ from the both sides of this equation. Noting the noncommutative rule (4.4), we have eq. (5.56). 
Note. The third term of eq. (5.56) is a total difference on the lattice and thus in fact satisfies the topological property (5.55):

$$
\varepsilon_{\mu \nu \rho \sigma} F_{\mu \nu}^{a}(n) F_{\rho \sigma}^{b}(n+\widehat{\mu}+\widehat{\nu})=4 \varepsilon_{\mu \nu \rho \sigma} \Delta_{\mu}\left[A_{\nu}^{a}(n) \Delta_{\rho} A_{\sigma}^{b}(n+\widehat{\nu})\right]
$$

This relation can easily be derived from the relation $F^{a} F^{b}=d\left(A^{a} F^{b}\right)$ which is valid in the context of noncommutative differential calculus. See ref. [32].

\section{Nontrivial anomalies in the abelian theory}

Because of the nilpotency $\delta_{B}^{2}=0$, any functional of the form $\mathcal{A}=\delta_{B} \mathcal{B}$ is a solution to eq. (1.3). If the functional $\mathcal{B}$ is local, such an anomaly can be removed by the redefinition of the effective action $\ln \operatorname{Det} M^{\prime} \rightarrow \ln \operatorname{Det} M^{\prime}-\mathcal{B}$ which does not change the physical content of the theory. Therefore the solution to the consistency condition (1.3) of the form $\mathcal{A}=\delta_{B} \mathcal{B}$ with a local functional $\mathcal{B}$ will be referred as trivial or BRS trivial.

\subsection{Nontrivial local SOlutions}

In this subsection, we study the structure of local solutions to the consistency condition (1.3) in the abelian theory $G=U(1)^{N}$. The BRS transformation is given by eq. (4.7). The ghost number of the solution is not restricted. We will find a very close analogue to the solutions in the continuum theory [16]. In the terminology of ref. [17], our result gives $\mathrm{H}(\delta \mid d)$ in abelian theories.

We seek the solution $\mathcal{A}$ by regarding $\mathcal{A}$ as a smooth and local functional of the gauge potential $A_{\mu}^{a}$ and the ghost field $c^{a}$. Since eq. (1.3) must hold for arbitrary configurations of $A_{\mu}^{a}$ and $c^{a}$, we have the variational equation

$$
\delta \delta_{B} \mathcal{A}=\sum_{n}\left\{\delta A_{\mu}^{a}(n) \delta_{B} \frac{\partial \mathcal{A}}{\partial A_{\mu}^{a}(n)}-\delta c^{a}(n)\left[\delta_{B} \frac{\partial \mathcal{A}}{\partial c^{a}(n)}+\Delta_{\mu}^{*} \frac{\partial \mathcal{A}}{\partial A_{\mu}^{a}(n)}\right]\right\}=0
$$

where $\delta \delta_{B} A_{\mu}^{a}=\Delta_{\mu} \delta c^{a}$ and $\delta \delta_{B} c^{a}=0$ have been used. The coefficients of the variations $\delta A_{\mu}^{a}(n)$ and $\delta c^{a}(n)$ have to vanish separately:

$$
\delta_{B} \frac{\partial \mathcal{A}}{\partial A_{\mu}^{a}(n)}=0, \quad \delta_{B} \frac{\partial \mathcal{A}}{\partial c^{a}(n)}+\Delta_{\mu}^{*} \frac{\partial \mathcal{A}}{\partial A_{\mu}^{a}(n)}=0
$$

Since $\mathcal{A}$ is local, $\partial \mathcal{A} / \partial A_{\mu}^{a}(n)$ is a local field. Then the abelian BRS cohomology (5.10) gives 
the general solution to the first equation with the ghost number $g$,

$$
\frac{\partial \mathcal{A}}{\partial A_{\mu}^{a}(n)}=c^{a_{1}}(n) \cdots c^{a_{g}}(n) \omega_{\mu}^{a\left[a_{1} \cdots a_{g}\right]}(n)+\delta_{B} Y_{\mu}^{a}(n),
$$

where $\omega_{\mu}^{a\left[a_{1} \cdots a_{g}\right]}$ depends only on the field strength. The second relation of eq. (6.2) then reads,

$$
\begin{gathered}
\delta_{B}\left[\frac{\partial \mathcal{A}}{\partial c^{a}(n)}+\Delta_{\mu}^{*} Y_{\mu}^{a}(n)\right]=-\Delta_{\mu}^{*}\left[c^{a_{1}}(n) \cdots c^{a_{g}}(n) \omega_{\mu}^{a\left[a_{1} \cdots a_{g}\right]}(n)\right] \\
=-c^{a_{1}}(n) \cdots c^{a_{g}}(n) \omega_{\mu}^{a\left[a_{1} \cdots a_{g}\right]}(n)+c^{a_{1}}(n-\widehat{\mu}) \cdots c^{a_{g}}(n-\widehat{\mu}) \omega_{\mu}^{a\left[a_{1} \cdots a_{g}\right]}(n-\widehat{\mu}) \\
=\delta_{B}\left[-\sum_{i=1}^{g}\left(\begin{array}{c}
g \\
i
\end{array}\right) A_{\mu}^{a_{1}}(n-\widehat{\mu}) \delta_{B} A_{\mu}^{a_{2}}(n-\widehat{\mu}) \cdots \delta_{B} A_{\mu}^{a_{i}}(n-\widehat{\mu})\right. \\
\left.\times c^{a_{i+1}}(n-\widehat{\mu}) \cdots c^{a_{g}}(n-\widehat{\mu}) \omega_{\mu}^{a\left[a_{1} \cdots a_{g}\right]}(n)\right] \\
-c^{a_{1}}(n-\widehat{\mu}) \cdots c^{a_{g}}(n-\widehat{\mu}) \Delta_{\mu}^{*} \omega_{\mu}^{a\left[a_{1} \cdots a_{g}\right]}(n)
\end{gathered}
$$

where we have used $c^{a}(n)=c^{a}(n-\widehat{\mu})+\delta_{B} A_{\mu}^{a}(n-\widehat{\mu})$ to pass from the second line to the third line. Considering the consistency of the above equation under $c^{a}(n) \rightarrow$ const., we have

$$
\Delta_{\mu}^{*} \omega_{\mu}^{a\left[a_{1} \cdots a_{g}\right]}(n)=0
$$

and then again from the BRS cohomology,

$$
\begin{gathered}
\frac{\partial \mathcal{A}}{\partial c^{a}(n)}=-\Delta_{\mu}^{*} Y_{\mu}^{a}(n)-\sum_{i=1}^{g}\left(\begin{array}{c}
g \\
i
\end{array}\right) A_{\mu}^{a_{1}}(n-\widehat{\mu}) \delta_{B} A_{\mu}^{a_{2}}(n-\widehat{\mu}) \cdots \delta_{B} A_{\mu}^{a_{i}}(n-\widehat{\mu}) \\
\times c^{a_{i+1}}(n-\widehat{\mu}) \cdots c^{a_{g}}(n-\widehat{\mu}) \omega_{\mu}^{a\left[a_{1} \cdots a_{g}\right]}(n) \\
+c^{a_{1}}(n) \cdots c^{a_{g-1}}(n) X^{a\left[a_{1} \cdots a_{g-1}\right]}(n)+\delta_{B} Y^{a}(n),
\end{gathered}
$$

where $X^{a\left[a_{1} \cdots a_{g-1}\right]}$ depends only on the field strength.

The functional $\mathcal{A}$ can be reconstructed from its variations (6.3) and (6.6) as follows. We introduce $\mathcal{A}_{t}$ by rescaling variables as $A_{\mu}^{a} \rightarrow t A_{\mu}^{a}$ and $c^{a} \rightarrow t c^{a}$. Noting $\mathcal{A}_{t=0}=0$ for $g>0$,

\footnotetext{
$\star$ For $g=0$, the following expressions hold by simply adding a constant $\mathcal{A}_{t=0}$.
} 
we have

$$
\mathcal{A}=\int_{0}^{1} d t \frac{\partial \mathcal{A}_{t}}{\partial t}=\int_{0}^{1} d t \sum_{n}\left[A_{\mu}^{a}(n) \frac{\partial \mathcal{A}_{t}}{\partial t A_{\mu}^{a}(n)}+c^{a}(n) \frac{\partial \mathcal{A}_{t}}{\partial t c^{a}(n)}\right] .
$$

After substituting eqs. (6.3) and (6.6) and shifting the coordinate $s \rightarrow s+\widehat{\mu}$, this yields

$$
\begin{aligned}
\mathcal{A}=\sum_{n}\{[ & A_{\mu}^{a_{0}}(n) c^{a_{1}}(n) \cdots c^{a_{g}}(n) \\
& \left.\quad-c^{a_{0}}(n+\widehat{\mu}) \sum_{i=1}^{g}\left(\begin{array}{c}
g \\
i
\end{array}\right) A_{\mu}^{a_{1}}(n) \delta_{B} A_{\mu}^{a_{2}}(n) \cdots \delta_{B} A_{\mu}^{a_{i}}(n) c^{a_{i+1}}(n) \cdots c^{a_{g}}(n)\right] \\
& \left.+c^{a_{1}}(n) \cdots c^{a_{g}}(n) \widetilde{X}^{\left[a_{1} \cdots a_{g}\right]}(n)\right\} \\
+ & \times \widetilde{\omega}_{\mu}^{a_{0}\left[a_{1} \cdots a_{g}\right]}(n) \\
& \delta_{B} \sum_{n}\left[A_{\mu}^{a}(n) \widetilde{Y}_{\mu}^{a}(n)-c^{a}(n) \widetilde{Y}^{a}(n)\right],
\end{aligned}
$$

where the following abbreviations have been introduced

$$
\begin{aligned}
& \widetilde{\omega}_{\mu}^{a\left[a_{1} \cdots a_{g}\right]}=\int_{0}^{1} d t t^{g} \omega_{\mu}^{a\left[a_{1} \cdots a_{g}\right]}{ }_{t}, \quad \tilde{X}^{\left[a_{1} \cdots a_{g}\right]}=\int_{0}^{1} d t t^{g-1} X^{\left[a_{1} \cdots a_{g}\right]} t \\
& \tilde{Y}_{\mu}^{a}=\int_{0}^{1} d t Y_{\mu}^{a}, \quad \tilde{Y}^{a}=\int_{0}^{1} d t Y^{a}{ }_{t} .
\end{aligned}
$$

Note that $\Delta_{\mu}^{*} \widetilde{\omega}_{\mu}^{a\left[a_{1} \cdots a_{g}\right]}=0$ from eq. (6.5) and that all these fields are local from the above construction. In particular, $\widetilde{\omega}_{\mu}^{a\left[a_{1} \cdots a_{g}\right]}$ and $\widetilde{X}^{\left[a_{1} \cdots a_{g}\right]}$ depend only on the field strength.

Eq. (6.8) provides the most general local solutions to the consistency condition. Yet it contains trivial solutions in various ways. First, by noting $c^{a_{0}}(n+\widehat{\mu})=c^{a_{0}}(n)+\delta_{B} A_{\mu}^{a_{0}}(n)$ and $\delta_{B} A_{\mu}^{a_{2}}(n)=c^{a_{2}}(n+\widehat{\mu})-c^{a_{2}}(n)$, it is easy to see that the symmetric part of $\widetilde{\omega}_{\mu}^{a_{0}\left[a_{1} \cdots a_{g}\right]}$ on $a_{0} \leftrightarrow a_{1}$ contributes only to a BRS trivial part:

$$
\begin{aligned}
& {\left[A_{\mu}^{a_{0}}(n) c^{a_{1}}(n) \cdots c^{a_{g}}(n)-g c^{a_{0}}(n+\widehat{\mu}) A_{\mu}^{a_{1}}(n) c^{a_{2}}(n) \cdots c^{a_{g}}(n)\right] \widetilde{\omega}_{\mu}^{\left(a_{0} a_{1}\right) a_{2} \cdots a_{g}}(n)} \\
& =\delta_{B}\left[-\frac{g}{2} A_{\mu}^{a_{0}}(n) A_{\mu}^{a_{1}}(n) c^{a_{2}}(n) \cdots c^{a_{g}}(n) \widetilde{\omega}_{\mu}^{\left(a_{0} a_{1}\right) a_{2} \cdots a_{g}}(n)\right],
\end{aligned}
$$

and

$$
\begin{aligned}
& c^{a_{0}}(n+\widehat{\mu}) A_{\mu}^{a_{1}}(n) \delta_{B} A_{\mu}^{a_{2}}(n) \cdots \delta_{B} A_{\mu}^{a_{i}}(n) c^{a_{i+1}}(n) \cdots c^{a_{g}}(n) \widetilde{\omega}_{\mu}^{\left(a_{0} a_{1}\right) a_{2} \cdots a_{g}}(n) \\
& =\delta_{B}\left[-\frac{1}{2} A_{\mu}^{a_{0}}(n) A_{\mu}^{a_{1}}(n) c^{a_{2}}(n) \delta_{B} A_{\mu}^{a_{3}}(n) \cdots \delta_{B} A_{\mu}^{a_{i}}(n) c^{a_{i+1}}(n) \cdots c^{a_{g}}(n) \widetilde{\omega}_{\mu}^{\left(a_{0} a_{1}\right) a_{2} \cdots a_{g}}(n)\right] .
\end{aligned}
$$


Therefore, for nontrivial solutions, we can assume that $\widetilde{\omega}_{\mu}^{a_{0}\left[a_{1} \cdots a_{g}\right]}$ is antisymmetric under the exchange $a_{0} \leftrightarrow a_{1}$, namely, $\widetilde{\omega}_{\mu}^{a_{0}\left[a_{1} \cdots a_{g}\right]}$ is totally antisymmetric $\widetilde{\omega}_{\mu}^{a_{0} \cdots a_{g}}=\widetilde{\omega}_{\mu}^{\left[a_{0} \cdots a_{g}\right]}$ in nontrivial solutions.

Henceforth we use the symbol $\simeq$ to indicate the equivalence relation modulo BRS trivial parts. The last term of eq. (6.8) is BRS trivial. Also, as noted above, $\widetilde{\omega}_{\mu}^{a_{0} \cdots a_{g}}$ is totally antisymmetric in nontrivial solutions. Then, by inserting $\delta_{B} A_{\mu}^{a_{j}}(n)=c^{a_{j}}(n+\widehat{\mu})-c^{a_{j}}(n)$ into eq. (6.8), and after some rearrangements, we have the following relatively simple expression

$$
\begin{gathered}
\mathcal{A} \simeq \sum_{n}\left[\sum_{k=0}^{g} c^{a_{1}}(n) \cdots c^{a_{k}}(n) A_{\mu}^{a_{0}}(n) c^{a_{k+1}}(n+\widehat{\mu}) \cdots c^{a_{g}}(n+\widehat{\mu}) \widetilde{\omega}_{\mu}^{\left[a_{0} \cdots a_{g}\right]}(n)\right. \\
\left.\quad+c^{a_{1}}(n) \cdots c^{a_{g}}(n) \widetilde{X}^{\left[a_{1} \cdots a_{g}\right]}(n)\right] .
\end{gathered}
$$

This expression takes a particularly simple form in terms of the noncommutative differential calculus. We introduce the dual 3 -form of $\widetilde{\omega}_{\mu}$ by

$$
\widetilde{\omega}_{\mu}^{\left[a_{0} \cdots a_{g}\right]}(n)=\frac{1}{3 !} \varepsilon_{\mu \nu \rho \sigma} \frac{(-1)^{g}}{g} \Omega_{\nu \rho \sigma}^{\left[a_{0} \cdots a_{g}\right]}(n+\widehat{\mu}) .
$$

Then by using the noncommutative rule (4.4), it is easy to see that

$$
\mathcal{A} d^{4} x(d \theta)^{g} \simeq \sum_{n}\left[\operatorname{sym}\left(A^{a_{0}} C^{a_{1}} \cdots C^{a_{g}}\right) \Omega^{\left[a_{0} \cdots a_{g}\right]}+C^{a_{1}} \cdots C^{a_{g}} \widetilde{X}^{\left[a_{1} \cdots a_{g}\right]} d^{4} x\right]
$$

On the other hand, the divergence-free condition (6.5) becomes

$$
d \Omega^{\left[a_{0} \cdots a_{g}\right]}=0
$$

We can now apply the covariant Poincaré lemma (5.11) to the 3 -form $\Omega^{\left[a_{0} \cdots a_{g}\right]}$ because it depends only on the field strength. This yields

$$
\Omega^{\left[a_{0} \cdots a_{g}\right]}=d \alpha_{2}^{\left[a_{0} \cdots a_{g}\right]}\left[\left\{F_{i}\right\}\right]+B_{3}^{\left[a_{0} \cdots a_{g}\right]}+F^{b} B_{1}^{\left[a_{0} \cdots a_{g}\right] b}
$$

and the contribution of $\alpha_{2}^{\left[a_{0} \cdots a_{g}\right]}$ can be absorbed into the second term of eq. (6.14) up to a 
trivial part, because

$$
\begin{aligned}
& \sum_{n} \operatorname{sym}\left(A^{a_{0}} C^{a_{1}} \cdots C^{a_{g}}\right) d \alpha_{2}^{\left[a_{0} \cdots a_{g}\right]} \\
& =\sum_{n}(-1)^{g} \operatorname{sym}\left(F^{a_{0}} C^{a_{1}} \cdots C^{a_{g}}\right) \alpha_{2}^{\left[a_{0} \cdots a_{g}\right]} \\
& \quad+s \sum_{n}\left[(-1)^{g+1} \frac{g}{2} \operatorname{sym}\left(A^{a_{0}} A^{a_{1}} C^{a_{2}} \cdots C^{a_{g}}\right) \alpha_{2}^{\left[a_{0} \cdots a_{g}\right]}\right] .
\end{aligned}
$$

Similarly, from the covariant Poincaré lemma, we have

$$
\begin{aligned}
& \widetilde{X}^{\left[a_{1} \cdots a_{g}\right]} d^{4} x \\
& =d \alpha_{3}^{\left[a_{1} \cdots a_{g}\right]}\left[\left\{F_{i}\right\}\right]+\mathcal{L}^{\left[a_{1} \cdots a_{g}\right]} d^{4} x+B_{4}^{\left[a_{1} \cdots a_{g}\right]}+F^{b} B_{2}^{\left[a_{1} \cdots a_{g}\right] b}+F^{b} F^{c} B_{0}^{\left[a_{1} \cdots a_{g}\right](b c)},
\end{aligned}
$$

and it is easy to see that $\alpha_{3}^{\left[a_{1} \cdots a_{g}\right]}$ does not contribute to the nontrivial part.

So, up to this stage, we have obtained

$$
\begin{aligned}
\mathcal{A} d^{4} x(d \theta)^{g} \simeq \sum_{n} & {\left[C^{a_{1}} \cdots C^{a_{g}} \mathcal{L}^{\left[a_{1} \cdots a_{g}\right]} d^{4} x\right.} \\
& +C^{a_{1}} \cdots C^{a_{g}}\left(B_{4}^{\left[a_{1} \cdots a_{g}\right]}+F^{b} B_{2}^{\left[a_{1} \cdots a_{g}\right] b}+F^{b} F^{c} B_{0}^{\left[a_{1} \cdots a_{g}\right](b c)}\right) \\
& \left.+\operatorname{sym}\left(A^{a_{0}} C^{a_{1}} \cdots C^{a_{g}}\right)\left(B_{3}^{\left[a_{0} \cdots a_{g}\right]}+F^{b} B_{1}^{\left[a_{0} \cdots a_{g}\right] b}\right)\right]
\end{aligned}
$$

where $\sum_{n} \delta \mathcal{L}^{\left[a_{1} \cdots a_{g}\right]} \neq 0$ under a certain local variation of the gauge potential. Formally this expression is identical to the list of nontrivial solutions in the continuum theory (see eq. (6.24) of the second reference of ref. [16]). Recall however that eq. (6.19) is an expression in the context of noncommutative differential calculus and that it is valid for a finite lattice spacing $a \neq 0$.

It is easy to see that eq. (6.19) satisfies $\delta_{B} \mathcal{A}=0$. Does eq. (6.19) not contain BRS trivial parts anymore? $\delta_{B}$ (something) is always proportional to a difference of the ghost field such as $\Delta_{\mu} c^{a}$. However, this does not necessarily imply that all terms of eq. (6.19) are BRS nontrivial. In contrast to the BRS cohomology (5.10), this expression contains the summation $\sum_{n}$. Therefore, after "integration by parts," a difference of ghost fields may result. 
In fact the term proportional to $B_{2}$ contains BRS trivial parts. Namely, by noting that $d C^{a}=-s A^{a}$, we have

$$
\begin{aligned}
\sum_{n} C^{a_{1}} \cdots C^{a_{g}} F^{b} B_{2}^{\left[a_{1} \cdots a_{g}\right] b} & \simeq \sum_{n} \operatorname{sym}\left(C^{a_{1}} \cdots C^{a_{g}} d A^{b}\right) B_{2}^{\left[a_{1} \cdots a_{g}\right] b} \\
& =\sum_{n}(-1)^{g} g \operatorname{sym}\left(s A^{a_{1}} C^{a_{2}} \cdots C^{a_{g}} A^{b}\right) B_{2}^{\left[a_{1} \cdots a_{g}\right] b} \\
& \simeq-\sum_{n} g \operatorname{sym}\left(A^{a_{1}} C^{a_{2}} \cdots C^{a_{g}} s A^{b}\right) B_{2}^{\left[a_{1} \cdots a_{g}\right] b} \\
& =\sum_{n}(-1)^{g} g \operatorname{sym}\left(s A^{b} C^{a_{2}} \cdots C^{a_{g}} A^{a_{1}}\right) B_{2}^{\left[a_{1} \cdots a_{g}\right] b} .
\end{aligned}
$$

Note that the commutator of $C^{a}$ and the field strength 2-form $F^{b}$ is proportional to a difference in the ghost field and thus, from the BRS cohomology, it is BRS trivial. Therefore the ordering of $C^{a}$ and $F^{b}$ is arbitrary in the first expression of eq. (6.20) up to BRS trivial parts. We have used this fact for the first $\simeq$ equality. By comparing the second line and the fourth line of the above expression, we see that eq. (6.20) is equivalent to

$$
\sum_{n} C^{b} C^{a_{2}} \cdots C^{a_{g}} F^{a_{1}} B_{2}^{\left[a_{1} \cdots a_{g}\right] b}=\sum_{n} C^{a_{1}} \cdots C^{a_{g}} F^{b} B_{2}^{\left[b a_{2} \cdots a_{g}\right] a_{1}}
$$

A comparison with the left hand side of eq. (6.20) shows that the antisymmetric part of $B_{2}^{\left[a_{1} \cdots a_{g}\right] b}$ under $a_{1} \leftrightarrow b$ is BRS trivial $\simeq 0$. Therefore $B_{2}^{\left[a_{1} \cdots a_{g}\right] b}$ must be symmetric under $a_{1} \leftrightarrow b$ to contribute nontrivial solutions.

Similarly, we have (suppressing $\sum_{n}$ )

$$
\begin{aligned}
C^{a_{1}} \cdots C^{a_{g}} F^{b} F^{c} B_{0}^{\left[a_{1} \cdots a_{g}\right](b c)} & \simeq \operatorname{sym}\left(C^{a_{1}} \cdots C^{a_{g}} F^{b}\right) F^{c} B_{0}^{\left[a_{1} \cdots a_{g}\right](b c)} \\
& \simeq C^{b} C^{a_{2}} \cdots C^{a_{g}} F^{a_{1}} F^{c} B_{0}^{\left[a_{1} \cdots a_{g}\right](b c)}
\end{aligned}
$$

and therefore $B_{0}^{\left[a_{1} \cdots a_{g}\right](b c)}$ must be symmetric under $a_{1} \leftrightarrow b$.

The term proportional to $B_{1}$ might also contain BRS trivial parts depending on symmetry of indices. However, the noncommutativity prevented us to imitate the procedure in the continuum theory [16].

Let us summarize the result: The general structure of local solutions to the consistency condition eq. (1.3) is given by eq. (6.19). The constant forms $B_{2}$ and $B_{0}$ have the following 
symmetries:

$$
B_{2}^{\left[a_{1} \cdots a_{g}\right] b}=B_{2}^{\left[b a_{2} \cdots a_{g}\right] a_{1}}, \quad B_{0}^{\left[a_{1} \cdots a_{g}\right](b c)}=B_{0}^{\left[b a_{2} \cdots a_{g}\right]\left(a_{1} c\right)} .
$$

The solution (6.19) is nontrivial, i.e., it cannot be written as $\mathcal{A}=\delta_{B} \mathcal{B}$ by using a local functional $\mathcal{B}$. The classical continuum limit of eq. (6.19) with eq. (6.23) coincides with the nontrivial solutions in the continuum theory [16] (with a partial exception for $B_{1}^{\left[a_{0} \cdots a_{g}\right] b}$ mentioned above). Then if eq. (6.19) was BRS trivial, the classical continuum limit of the local functional $\mathcal{B}$ would act as a counter term for the nontrivial solutions in the continuum theory. But this contradicts with the result of ref. [16].

In this subsection, we have obtained the general nontrivial local solutions with an arbitrary ghost number. For discussions of the gauge anomaly in the next subsection, knowledge of solutions with ghost number one is enough. The solutions with higher ghost number, however, might become relevant for future applications. For example, it might be possible to address the commutator anomaly [61] in the context of lattice gauge theory starting with the above expressions.

\subsection{Gauge anomaly in abelian theory}

If we restrict solutions with the ghost number unity, eq. (6.19) tells us that

$$
\begin{aligned}
\mathcal{A} \simeq \sum_{n}\{ & c^{a}(n) \mathcal{L}^{a}(n) \\
& +c^{a}(n)\left[\alpha^{a}+\beta_{[\mu \nu]}^{(a b)} F_{\mu \nu}^{b}(n)+\gamma^{(a b c)} \varepsilon_{\mu \nu \rho \sigma} F_{\mu \nu}^{b}(n) F_{\rho \sigma}^{c}(n+\widehat{\mu}+\widehat{\nu})\right] \\
& \left.+\left[A_{\mu}^{a}(n) c^{b}(n+\widehat{\mu})+c^{b}(n) A_{\mu}^{a}(n)\right]\left[f_{\mu}^{[a b]}+g_{[\mu \nu \rho]}^{[a b] c} F_{\nu \rho}^{c}(n+\widehat{\mu})\right]\right\},
\end{aligned}
$$

where we have used the noncommutative rule (4.4) and the symmetry of indices (6.23). In this expression, the function $\mathcal{L}^{a}(n)$ satisfies $\sum_{n} \delta \mathcal{L}^{a}(n) \neq 0$ for a certain local variation.

Eq. (6.24) provides the general candidate of nontrivial local gauge anomalies in the abelian theory $G=U(1)^{N}$. However, depending on the situation, we may further restrict the coefficients in various ways.

\footnotetext{
$\star$ The information about these is important [16] when one explicitly considers the higher order sequence $\mathcal{A}_{\ell}$ with $\ell \geq 4$ for the nonabelian anomaly; we do not pursue this in this paper.
} 
(1) When $G=U(1)$, the last line vanishes due to the anti-symmetrization of indices. Eq. (6.24) then reproduces Lüscher's result (1.1) except for the "non-topological term" $\mathcal{L}^{a}$.

(2) The non-topological term $\mathcal{L}^{a}$ and the term proportional to $f_{\mu}^{[a b]}$ do not appear, if the anomaly has the topological property $\delta \mathcal{A}[c, A]=0$ for $c^{a}(n) \rightarrow$ const., where $\delta$ is an arbitrary local variation of the gauge potential.

(3) If the couplings of the Weyl fermion to gauge fields have the same structure for all $U(1)$ factors except coupling constants (practically this is always the case), then all the coefficients are independent of group indices and we have $\alpha^{a} \rightarrow \alpha, \beta_{[\mu \nu]}^{(a b)} \rightarrow \beta_{[\mu \nu]}, \gamma^{(a b c)} \rightarrow \gamma, f_{\mu}^{[a b]} \rightarrow 0$, $g_{[\mu \nu \rho]}^{[a b] c} \rightarrow 0$.

(4) From the dimension counting, all the terms except $\mathcal{L}^{a}$ and the term proportional to $\gamma^{(a b c)}$ have negative powers of the lattice spacing as the overall coefficient. Therefore, if the classical continuum limit $a \rightarrow 0$ of $\mathcal{A}$ is finite (for a smooth background), all the terms except $\mathcal{L}^{a}$ and $\gamma^{(a b c)}$ must be absent. In particular, if $\lim _{a \rightarrow 0} \mathcal{A}$ reproduces the gauge anomaly in the continuum theory, then $\gamma^{(a b c)}=-\epsilon_{H} /\left(96 \pi^{2}\right)$ for a single Weyl fermion.

Let us assume that (2) and (4) hold. Then we have a content of the theorem for the abelian gauge theory which we stated in section 3 .

\section{Nonabelian extension}

In this section, we study the gauge anomaly for a general (compact) gauge group $G=$ $\prod_{\alpha} G_{\alpha}$, where $G_{\alpha}$ is a simple group or a $U(1)$ factor. The candidate of the anomaly is given by the solution with ghost number one to the consistency condition (1.3). The general solution to eq. (1.3) is expressed as

$$
\mathcal{A}=\widetilde{\mathcal{A}}+\delta_{B} \mathcal{B}
$$

where $\widetilde{\mathcal{A}} \neq \delta_{B} \mathcal{B}$ is the BRS nontrivial part ( $\mathcal{B}$ is a local functional). The BRS transformation is given by eq. (1.2) and it takes the following form in terms of the gauge potential

$$
\delta_{B} A_{\mu}(n)=\frac{1}{2} A_{\mu}(n) \wedge\left[\operatorname{coth} \frac{1}{2} A_{\mu}(n) \wedge \Delta_{\mu} c(n)+c(n)+c(n+\widehat{\mu})\right],
$$

where $X \wedge Y=[X, Y], X^{2} \wedge Y=[X,[X, Y]]$ and so on, and $1 \wedge Y=Y$ is understood. We shall use both the matrix notation $A_{\mu}(n)$ and $c(n)$, and the component notation $A_{\mu}(n)=$ $\sum_{s} A_{\mu}^{a}(n) T^{a}$ and $c(n)=\sum_{a} c^{a}(n) T^{a}$. 
To make our problem tractable, we make the following assumptions about the anomaly $\mathcal{A}$.

(I) $\mathcal{A}$ is a smooth and local functional of the gauge potential $A_{\mu}$ and the ghost field $c$.

(II) The classical continuum limit of $\mathcal{A}$ reproduces the anomaly in the continuum theory as in eq. (2.28).

(III) $U(1)$ gauge anomalies in $\mathcal{A}$ have the topological property as in eq. (3.3).

Under these assumptions, in section 7.2 , we show that the anomaly $\mathcal{A}$, if it exists, is unique (up to the BRS trivial part) to all orders of the gauge potential. The unique anomaly is proportional to the gauge anomaly in the continuum theory and this establishes the theorem for nonabelian theories, stated in section 3. In section 7.3, we show that such a solution in fact exists. As a preparation for sec. 7.2, we need the following lemma.

\subsection{Basic lemma: Adjoint invariance}

Adjoint invariance. Without loss of generality, one can assume that a nontrivial local solution $\widetilde{\mathcal{A}}$ is invariant under the adjoint transformation

$$
\delta^{a} \widetilde{\mathcal{A}}=0,
$$

where the adjoint transformation $\delta^{a}$ is defined by

$$
\delta^{a} U(n, \mu)=\left[T^{a}, U(n, \mu)\right], \quad \delta^{a} A_{\mu}^{b}(n)=-i f^{a b c} A_{\mu}^{c}(n), \quad \delta^{a} c^{b}(n)=-i f^{a b c} c^{c}(n) .
$$

Note. There is freedom to add a BRS trivial part $\delta_{B} \mathcal{B}$ to a nontrivial solution. The above lemma asserts that it is always possible to choose $\mathcal{B}$ such that $\widetilde{\mathcal{A}}$ is adjoint invariant. The adjoint transformation $\delta^{a}$ satisfies the following relations

$$
\left[\delta_{B}, \delta^{a}\right]=0, \quad\left[\Delta_{\mu}^{*}, \delta^{a}\right]=0, \quad\left[\delta^{a}, \delta^{b}\right]=i f^{a b c} \delta^{c} .
$$

Proof. The functional $\widetilde{\mathcal{A}}$ is local, i.e., the field $\widetilde{a}(n)$ in $\widetilde{\mathcal{A}}=\sum_{n} \widetilde{a}(n)$ is a local field. We express $\widetilde{a}(n)$ in terms of the following set of variables, which was introduced in the proof of 
the abelian BRS cohomology in ref. [32]:

$$
A_{i}^{a}=\left(\Delta_{1}\right)^{p_{1}} \cdots\left(\Delta_{\mu}\right)^{p_{\mu}} A_{\mu}^{a}(n), \quad F_{i}^{a}=\left(\Delta_{1}\right)^{p_{1}} \cdots\left(\Delta_{D}\right)^{p_{D}} F_{\mu \nu}^{a}(n),
$$

for the gauge potential ( $D$ is the dimension of the lattice) and

$$
c_{i}^{a}=\delta_{0} A_{i}^{a}=\left(\Delta_{1}\right)^{p_{1}} \cdots\left(\Delta_{\mu}\right)^{p_{\mu}} \Delta_{\mu} c^{a}(n), \quad \text { and } \quad c^{a}(n),
$$

for the ghost field; here $\delta_{0}$ is the abelian BRS transformation, $\delta_{0} A_{\mu}^{a}(n)=\Delta_{\mu} c^{a}(n)$ and $\delta_{0} c^{a}(n)=0$. In these expressions, the symbol $\left(\Delta_{\mu}\right)^{p}$ ( $p$ is an integer) has been defined by

$$
\left(\Delta_{\mu}\right)^{p}= \begin{cases}\Delta_{\mu}^{p}, & \text { for } p>0 \\ 1, & \text { for } p=0 \\ \Delta_{\mu}^{*-p}, & \text { for } p<0\end{cases}
$$

Then it can be shown [32] that these variables, $A_{i}^{a}, F_{i}^{a}, c_{i}^{a}$ and $c^{a}(n)$ span a (over)complete set, i.e., the field $\widetilde{a}(n)$ can be expressed as a function of these variables. A little thought shows that the relation

$$
\left[\frac{\partial}{\partial c^{a}(n)}, \Delta_{\mu}^{*}\right]=0
$$

holds for arbitrary functions of these variables.

Since the nonabelian BRS transformation (1.2) or (7.2) has the structure,

$$
\begin{aligned}
& \delta_{B} A_{\mu}^{a}(n)=i f^{a b c} A_{\mu}^{b}(n) c^{c}(n)+\left(\text { terms proportional to } \Delta_{\mu} c^{a}\right), \\
& \delta_{B} c^{a}(n)=-\frac{1}{2} i f^{a b c} c^{b}(n) c^{c}(n),
\end{aligned}
$$

we have

$$
\delta_{B}\left\{\begin{array}{c}
A_{i}^{a} \\
F_{i}^{a} \\
c_{i}^{a}
\end{array}\right\}=-c^{b}(n) \delta^{b}\left\{\begin{array}{c}
A_{i}^{a} \\
F_{i}^{a} \\
c_{i}^{a}
\end{array}\right\}+\left(\text { terms proportional to } c_{i}^{a}\right)
$$

and thus

$$
\delta^{a}=-\left\{\delta_{B}, \frac{\partial}{\partial c^{a}(n)}\right\}
$$

on functions of the variables $A_{i}^{a}, F_{i}^{a}, c_{i}^{a}$ and $c^{a}(n)$ (for $c^{a}(n)$ this follows from eq. (7.10)). 
The remaining argument to prove the lemma is almost identical to that of ref. [16]. We introduce the Casimir operator:

$$
\mathcal{O}_{K}=g^{a_{1} \cdots a_{m(K)}} \delta^{a_{1}} \cdots \delta^{a_{m(K)}}
$$

where $g^{a_{1} \cdots a_{m(K)}}=\operatorname{str} T^{a_{1}} \cdots T^{a_{m(K)}}$ are totally symmetric constants and $K$ runs from 1 to the rank of the semisimple part of the group $G$ [62]. Using the completeness of eigenfunctions of $\mathcal{O}_{K}$, we decompose $\widetilde{a}(n)$ according to the representation $\lambda, \widetilde{a}(n)=\sum_{\lambda} \widetilde{a}^{\lambda}(n)$, where

$$
\mathcal{O}_{K} \widetilde{a}^{\lambda}(n)=k(K, \lambda) \widetilde{a}^{\lambda}(n)
$$

and $k(K, \lambda)$ is the eigenvalue. Since $\delta_{B} \widetilde{\mathcal{A}}=\sum_{n} \delta_{B} \widetilde{a}(n)=0$, the dual of the algebraic Poincaré lemma (5.1) $(p=D)$ shows that

$$
\delta_{B} \widetilde{a}(n)=\Delta_{\mu}^{*} X_{\mu}(n)
$$

where $X_{\mu}(n)$ is a local field. We again apply to this equation the decomposition similar to eq. (7.14):

$$
\delta_{B} \widetilde{a}^{\lambda}(n)=\Delta_{\mu}^{*} X_{\mu}^{\lambda}(n)
$$

where use of relations (7.5) has been made.

Now suppose that there exist $K$ and $\lambda$ such that $k(K, \lambda) \neq 0$ in eq. (7.14). Then, by using eqs. (7.13), (7.12), (7.5) and (7.16), we have

$$
\begin{aligned}
\widetilde{a}^{\lambda}(n)=\delta_{B} & \frac{-1}{k(K, \lambda)} g^{a_{1} \cdots a_{m(K)}} \delta^{a_{m(K)}} \cdots \delta^{a_{2}} \frac{\partial}{\partial c^{a_{1}}(n)} \widetilde{a}^{\lambda}(n) \\
& +\Delta_{\mu}^{*} \frac{-1}{k(K, \lambda)} g^{a_{1} \cdots a_{m(K)}} \delta^{a_{m(K)}} \cdots \delta^{a_{2}} \frac{\partial}{\partial c^{a_{1}}(n)} X_{\mu}^{\lambda}(n)
\end{aligned}
$$

Namely, $\widetilde{\mathcal{A}}$ contains a BRS trivial part $\sum_{n} \widetilde{a}^{\lambda}(n)$ which we can remove by $\delta_{B} \mathcal{B}$. After repeating this procedure, all the eigenvalues $k(K, \lambda)$ in eq. (7.14) are made to vanish and this implies that $\lambda$ is the singlet representation. Therefore, we can always assume that a BRS nontrivial solution is adjoint invariant, $\delta^{a} \widetilde{\mathcal{A}}=0$. 
We next derive a constraint for $\widetilde{\mathcal{A}}$ following from the assumption (III) made above and the lemma (7.3). Set $c^{a}(n) \rightarrow c^{a}=$ const. Since the ghost number of $\widetilde{\mathcal{A}}$ is unity, we can write it as

$$
\widetilde{\mathcal{A}}=c^{a} k^{a \lambda} X^{\lambda}[A]
$$

where $k^{a \lambda}$ are constants and $\lambda$ labels the linearly independent functional $X^{\lambda}[A]$. We then consider the following two cases separately:

(1) When the index $a$ of the ghost field in eq. (7.18) belongs to a $U(1)$ factor group $U(1)_{\alpha}$, we have

$$
\widetilde{\mathcal{A}}=c^{U(1)_{\alpha}} X[A]
$$

However, from assumption (III), we have $\delta X=0$, where $\delta$ is an arbitrary local variation of the gauge potential.

(2) When the index $a$ of the ghost field in eq. (7.18) belongs to a simple group, the BRS transformation (1.2) or (7.2) becomes for $c^{a}(x) \rightarrow c^{a}=$ const.,

$$
\begin{aligned}
& \delta_{B} A_{\mu}^{a}(n)=-i f^{a b c} c^{b} A_{\mu}^{c}(n)=-c^{b} \delta^{b} A_{\mu}^{a}(n), \\
& \delta_{B} c^{a}=-\frac{1}{2} i f^{a b c} c^{b} c^{c}=-\delta_{B} c^{a}-c^{b} \delta^{b} c^{a}
\end{aligned}
$$

where $\delta^{a}$ is the adjoint transformation. But since the lemma (7.3) asserts that $\delta^{a} \widetilde{\mathcal{A}}=0$, the consistency condition becomes for $c^{a}(n) \rightarrow$ const.,

$$
\delta_{B} \widetilde{\mathcal{A}}=-\delta_{B}\left(c^{a} k^{a \lambda}\right) X^{\lambda}[A]=0 .
$$

This requires $\delta_{B}\left(c^{a} k^{a \lambda}\right)=0$. Then the Lie algebra cohomology in ref. [16] asserts that $c^{a} k^{a \lambda}=\operatorname{tr} c=0$ for a simple group. This shows that $\tilde{\mathcal{A}}=0$ for $c^{a}(n) \rightarrow$ const. This conclusion might be dangerous because there is a possibility of having a total divergence. To avoid this, it is enough to consider a local variation which implies $\delta X^{\lambda}=0$ where $\delta$ is an arbitrary local variation of the gauge potential.

\footnotetext{
$\star$ Note that the addition of $\delta_{B} \mathcal{B}$ with a local term $\mathcal{B}$ does not influence the topological property, as noted in sec. 2 .
} 
Combining above (1) and (2), we see that the assumption (III) implies

$$
\delta \widetilde{\mathcal{A}}=0, \quad \text { for } \quad c^{a}(n) \rightarrow \text { const. },
$$

where $\delta$ is an arbitrary local variation of the gauge potential. This provides a strong constraint for the possible form of $\widetilde{\mathcal{A}}$, as will be seen in the next subsection.

\subsection{UNIQUENESS OF THE NONTRIVIAL ANOMALY}

We now expand the anomaly $\mathcal{A}(7.1)$ in powers of the gauge potential as

$$
\mathcal{A}=\sum_{\ell=1}^{\infty} \mathcal{A}_{\ell}, \quad \widetilde{\mathcal{A}}=\sum_{\ell=1}^{\infty} \widetilde{\mathcal{A}}_{\ell}, \quad \mathcal{B}=\sum_{\ell=1}^{\infty} \mathcal{B}_{\ell},
$$

where $\ell$ stands for the number of powers of $c$ and $A_{\mu}$ (recall that the ghost number of $\mathcal{A}$ is unity). We decompose also the BRS transformation (7.2) according to powers of the fields, $\delta_{B}=\sum_{\ell=0}^{\infty} \delta_{\ell}$, where

$$
\begin{aligned}
& \delta_{0} A_{\mu}(n)=\Delta_{\mu} c(n), \quad \delta_{0} c(n)=0, \\
& \delta_{1} A_{\mu}(n)=\frac{1}{2}\left[A_{\mu}(n), c(n)+c(n+\widehat{\mu})\right], \quad \delta_{1} c(n)=-c(n)^{2}, \\
& \left.\left.\delta_{2 k} A_{\mu}(n)=(-1)^{k-1} \frac{B_{k}}{(2 k) !}[\underbrace{A_{\mu}(n),\left[A_{\mu}(n), \cdots,\left[A_{\mu}(n)\right.\right.}_{2 k}, \Delta_{\mu} c(n)] \cdots\right]\right], \\
& \delta_{2 k} c(n)=0, \quad \text { for } \quad k \geq 1 .
\end{aligned}
$$

Here $B_{k}$ is the Bernoulli number, and $\delta_{2 k+1}=0$ for $k \geq 1$. Note that, in terms of components $A_{\mu}^{a}$ and $c^{a}, \delta_{0}$ has an identical form to the abelian BRS transformation (4.7). The nilpotency $\delta_{B}^{2}=0$ implies

$$
\sum_{k=0}^{\ell} \delta_{k} \delta_{\ell-k}=0, \quad \text { for } \quad \ell \geq 0,
$$

and the consistency condition (1.3) takes the form

$$
\delta_{0} \widetilde{\mathcal{A}}_{\ell}=-\sum_{k=1}^{\ell-1} \delta_{k} \widetilde{\mathcal{A}}_{\ell-k}, \quad \text { for } \quad \ell \geq 1 .
$$

Since

$$
\mathcal{A}_{\ell}=\widetilde{\mathcal{A}}_{\ell}+\sum_{k=0}^{\ell-1} \delta_{k} \mathcal{B}_{\ell-k},
$$

if $\widetilde{\mathcal{A}}_{\ell}$ contains $\delta_{0}$-trivial part $\delta_{0} \mathcal{B}_{\ell}^{\prime}, \mathcal{B}_{\ell}^{\prime}$ can always be absorbed into $\mathcal{B}_{\ell}$. Therefore we can 
assume that

$$
\delta_{0} \mathcal{B}_{\ell}^{\prime} \text { in } \widetilde{\mathcal{A}}_{\ell} \text { can always be neglected. }
$$

The constraint (7.22) has to hold for each order:

$$
\delta \widetilde{\mathcal{A}}_{\ell}=0, \quad \text { for } \quad c^{a}(n) \rightarrow \text { const. }
$$

where $\delta$ is an arbitrary local variation of the gauge potential. Also the correct classical continuum limit (II) requires

$$
\widetilde{\mathcal{A}}_{\ell} \stackrel{a \rightarrow 0}{\rightarrow} O\left(A^{\ell-1}\right) \text { term of eq. (2.28). }
$$

We consider the local solution to the consistency condition (7.26) which satisfies the conditions (7.29) and (7.30), order by order. The first equation in eq. (7.26) is

$$
\delta_{0} \widetilde{\mathcal{A}}_{1}=0
$$

This equation is completely identical to the consistency condition in the abelian theory. Therefore, from our result in the preceding section, eq. (6.24), the general form of $\widetilde{\mathcal{A}}_{1}$ which satisfies eqs. (7.29) and (7.30) is given by

$$
\widetilde{\mathcal{A}}_{1}=0
$$

where we have noted eq. (7.28). The next equation in eq. (7.26) is

$$
\delta_{0} \widetilde{\mathcal{A}}_{2}=0
$$

Again from the result in the abelian theory (6.24), we have

$$
\widetilde{\mathcal{A}}_{2}=0
$$

where use of eqs. (7.28)-(7.30) has been made to conclude this. 
The solution to the next equation

$$
\delta_{0} \widetilde{\mathcal{A}}_{3}=0
$$

has a $\delta_{0}$-nontrivial part. From eq. (6.24), and from eqs. (7.28)-(7.30), we have

$$
\begin{aligned}
\widetilde{\mathcal{A}}_{3}=-\frac{\epsilon_{H}}{24 \pi^{2}} \sum_{n}\{ & \varepsilon_{\mu \nu \rho \sigma} \frac{1}{2} \operatorname{tr} c^{(\alpha)}(n) \Delta_{\mu}\left\{A_{\nu}^{(\alpha)}(n), \Delta_{\rho} A_{\sigma}^{(\alpha)}(n+\widehat{\nu})\right\} \\
& +\varepsilon_{\mu \nu \rho \sigma} c^{U(1)_{\beta}}(n) \Delta_{\mu}\left[A_{\nu}^{U(1)_{\beta}}(n) \Delta_{\rho} A_{\sigma}^{U(1)_{\beta}}(n+\widehat{\nu})\right] \\
& +\varepsilon_{\mu \nu \rho \sigma} c^{U(1)_{\beta}}(n) \operatorname{tr} \Delta_{\mu}\left[A_{\nu}^{(\alpha)}(n) \Delta_{\rho} A_{\sigma}^{(\alpha)}(n+\widehat{\nu})\right] \\
& +\varepsilon_{\mu \nu \rho \sigma} \operatorname{tr} c^{(\alpha)}(n) \Delta_{\mu}\left[A_{\nu}^{U(1)_{\beta}}(n) \Delta_{\rho} A_{\sigma}^{(\alpha)}(n+\widehat{\nu})\right] \\
& \left.+\varepsilon_{\mu \nu \rho \sigma} \operatorname{tr} c^{(\alpha)}(n) \Delta_{\mu}\left[A_{\nu}^{(\alpha)}(n) \Delta_{\rho} A_{\sigma}^{U(1)_{\beta}}(n+\widehat{\nu})\right]\right\},
\end{aligned}
$$

where we have used relation (5.58) to make the property (7.29) manifest. Note that the condition (7.29) is crucial to eliminate the possibility that the $\mathcal{L}^{a}$ term in eq. (6.24) appears.

The next equation in eq. (7.26) is

$$
\delta_{0} \widetilde{\mathcal{A}}_{4}=-\delta_{1} \widetilde{\mathcal{A}}_{3}
$$

The solution to this equation $\widetilde{\mathcal{A}}_{4}$, if it exists, is unique. If another $\widetilde{\mathcal{A}}_{4}^{\prime}$ which also satisfies the conditions (7.29) and (7.30) exists, then

$$
\delta_{0}\left(\widetilde{\mathcal{A}}_{4}^{\prime}-\widetilde{\mathcal{A}}_{4}\right)=0
$$

and the quantity inside the brackets again satisfies eq. (7.29). Eqs. (7.28) and (6.24) then imply that $\widetilde{\mathcal{A}}_{4}^{\prime}-\widetilde{\mathcal{A}}_{4}=0$.

The above argument can be repeated for higher $\widetilde{\mathcal{A}}_{\ell}$ 's. Suppose that a sequence for the nontrivial part, $\widetilde{\mathcal{A}}_{3}, \widetilde{\mathcal{A}}_{4}, \ldots, \widetilde{\mathcal{A}}_{\ell-1}$, has been obtained. Then the next term $\widetilde{\mathcal{A}}_{\ell}$ has to satisfy eq. (7.26) and the conditions (7.29) and (7.30). Then the same argument as above shows that the solution $\widetilde{\mathcal{A}}_{\ell}$, if it exists, is unique.

We have seen that the sequence $\widetilde{\mathcal{A}}_{\ell}$ for the nontrivial anomaly $\widetilde{\mathcal{A}}$, which satisfies the assumptions (I), (II) and (III), if it exists, is unique up to a BRS trivial part. There is 
no free parameter which can appear in higher $\widetilde{\mathcal{A}}_{\ell}$ 's. Moreover, this uniqueness shows that the anomaly cancellation in the continuum theory implies that of the lattice theory: If the first nontrivial term $\widetilde{\mathcal{A}}_{3}(7.36)$, which is proportional to the anomaly in the continuum theory $\operatorname{tr}_{R-L} T^{a}\left\{T^{b}, T^{c}\right\}$ etc., is canceled among the fermion multiplet, then the subsequent sequence of $\widetilde{\mathcal{A}}_{\ell}$ for $\ell \geq 4$ is completely canceled. In other words, the possible nontrivial local anomaly on the lattice, $\widetilde{\mathcal{A}}$, under the assumptions (I)-(III), is always proportional to the anomaly in the continuum theory, to all orders in powers of the gauge potential. This establishes our theorem for nonabelian theories, stated in section 3.

The existence of the nontrivial sequence $\widetilde{\mathcal{A}}_{\ell}$ for $\ell \geq 4$ might be examined by repeatedly solving eq. (7.26). However, eq. (7.24) suggests that the explicit form of higher $\widetilde{\mathcal{A}}_{\ell}$ 's will become quite complicated as $\ell$ increases. In the next subsection, instead of this analysis, we will give a "compact" form of the nontrivial solution which manifestly satisfies eq. (7.22) and the assumptions (I) (at least for the perturbative region (2.9)) and (II). This explicitly shows the existence of the nontrivial sequence, $\widetilde{\mathcal{A}}_{\ell}$ with $\ell \geq 4$. To write down the compact solution, however, we need the interpolation technique of lattice fields with which the BRS transformation takes a quite simple form. Therefore, we give a quick summary of the method of ref. [34] in the first part of the next subsection.

\subsection{COMPACT FORM OF THE NONTRIVIAL ANOMALY}

First we recapitulate the essence of the interpolation method of lattice fields in ref. [34] (simply extended for infinite lattices). ${ }^{\star}$ For our argument, the interpolation method has to possess several properties which we will verify. To distinguish from the fields defined on lattice sites $n$, we use the continuous coordinate $x$ for interpolated fields.

The method of ref. [34] consists of the following two steps.

Step 1. One first constructs the interpolated gauge potential $A_{\mu}^{(m)}(x)$ within each hypercube $h(m)$, here $m$ stands for the origin of the hypercube, such that the gauge potentials in neighboring hypercubes $h(m-\widehat{\mu})$ and $h(m)$ are related by the transition function $v_{m, \mu}(x)$ of Lüscher's principal fiber bundle [40],

$$
A_{\lambda}^{(m-\widehat{\mu})}(x)=v_{m, \mu}(x)\left[\partial_{\lambda}+A_{\lambda}^{(m)}(x)\right] v_{m, \mu}(x)^{-1}
$$

* Under the same conditions we will assume, the method of ref. [35] might be adopted as well. 
on the intersection of the two hypercubes $x \in h(m-\widehat{\mu}) \cap h(m)$ (which is a 3-dimensional cube). For the transition function $v_{m, \mu}(x)$ to be well-defined, the gauge field configuration must be "non-exceptional" [40]. As already noted, it can be shown that if $\epsilon$ in eq. (2.1) is sufficiently small, the gauge field configuration is non-exceptional. So we assume that $\epsilon$ has been chosen so that this is the case. The gauge potential which satisfies eq. (7.39) can be constructed, starting with a special gauge $A_{\lambda}^{(m)}(x)=0$ at $x \sim m$. The explicit expression of $A_{\lambda}^{(m)}(x)$ in terms of $v_{m, \mu}(x)$, which is eventually expressed by the link variables $U$ [40], is given in ref. [34]. We do not reproduce it here because it is rather involved and we do not need the explicit form in what follows. The interesting property of $A_{\mu}^{(m)}(x)$ is [34]

$$
\mathcal{P} \exp \left[\int_{0}^{1} d t A_{\mu}^{(m)}(n+(1-t) \widehat{\mu})\right]=u_{n, n+\widehat{\mu}}^{m} .
$$

Namely, the Wilson line constructed from the interpolated gauge potential $A_{\mu}^{(m)}(x)$ coincides with the link variable in the complete axial gauge of ref. [40].

Step 2.1. The section of the principal fiber bundle [40]

$$
w^{m}(n)=U(m, 1)^{z_{1}} U\left(m+z_{1} \widehat{1}, 2\right)^{z_{2}} U\left(m+z_{1} \widehat{1}+z_{2} \widehat{2}, 3\right)^{z_{3}} U\left(m+z_{1} \widehat{1}+z_{2} \widehat{2}+z_{3} \widehat{3}, 4\right)^{z_{4}} \in G,
$$

is defined for each lattice site $n$ belonging to the hypercube $h(m)$ where $n=m+\sum_{\mu} z_{\mu} \widehat{\mu}$. This section is then smoothly interpolated, first on the links, next on the plaquettes, on the cubes, and finally inside the hypercube $h(m)$. At this stage, if the homotopy group $\pi_{M-1}(G)$ is nontrivial, the smooth interpolation of the section $w^{m}(x)$ into a $M$-dimensional (sub)lattice may fail, depending on the configuration of the section $w^{m}(x)$ on a boundary of the $M$ dimensional (sub)lattice. For example, for $G=U(1), \pi_{1}(U(1))=Z$, and if the local winding of $w^{m}(x)$ around a boundary of the plaquette $p(m, \mu, \nu)$,

$$
Q(m, \mu, \nu)=\frac{i}{2 \pi} \int_{\partial p(m, \mu, \nu)} d x_{\mu} \varepsilon_{\mu \nu} w^{m}(x)^{-1} \partial_{\nu} w^{m}(x)
$$

does not vanish, then the interpolation of the section $w^{m}(x)$ into the plaquette $p(m, \mu, \nu)$ develops a singularity. Similarly, for $G=S U(2), \pi_{3}(S U(2))=Z$, and the local winding is 
given $\mathrm{by}^{\dagger}$

$$
Q(m)=\frac{1}{24 \pi^{2}} \int_{\partial h(m)} d^{3} x_{\mu} \varepsilon_{\mu \nu \rho \sigma} \operatorname{tr} w^{m}(x)^{-1} \partial_{\nu} w^{m}(x) w^{m}(x)^{-1} \partial_{\rho} w^{m}(x) w^{m}(x)^{-1} \partial_{\sigma} w^{m}(x) .
$$

If $Q(m)$ does not vanish, then the interpolation of $w^{m}(x)$ into the hypercube $h(m)$ develops the singularity. If these singularities arise, the description in term of the interpolated fields becomes inadequate. $\ddagger$ Fortunately, all local windings vanish within the perturbative region (2.9), for sufficiently small $\epsilon$. If $\epsilon$ in (2.9) is sufficiently small, the norm of the exponent of a product of several link variables is also small, and the expression of the interpolated section [34] cannot have the "jump" on a boundary of the $M$-dimensional (sub)lattice. This implies that there is no local winding.

Step 2.2. With the smooth interpolated section $w^{m}(x)$, we define the "global" interpolated gauge potential by

$$
A_{\lambda}(x)=w^{m}(x)^{-1}\left[\partial_{\lambda}+A_{\lambda}^{(m)}(x)\right] w^{m}(x),
$$

for $x \in h(m)$. The resulting interpolated gauge potential $A_{\lambda}(x)$ is Lie algebra valued.

Now, we need the following properties of the interpolation method to express the nontrivial local solution.

(i) The gauge covariance. This is the most important property for our purpose. Namely, there exists a smooth interpolation of the gauge transformation (in our present context this becomes a smooth interpolation of the ghost field) and the lattice gauge (BRS) transformation on the link variables takes an identical form as that of the continuum theory. This property was shown in ref. [34]. Therefore, the BRS transformation (1.2) induces

$$
\delta_{B} A_{\mu}^{a}(x)=\partial_{\mu} c^{a}(x)+i f^{a b c} A_{\mu}^{b}(x) c^{c}(x), \quad \delta_{B} c^{a}(x)=-\frac{1}{2} i f^{a b c} c^{b}(x) c^{c}(x),
$$

on the interpolated fields.

$\dagger$ The total winding $Q=\sum_{m} Q(m)$ on a finite periodic lattice is nothing but Lüscher's topological charge [40].

$\ddagger$ The procedure of ref. [35] can avoid this difficulty. 
(ii) The transverse continuity. This means that the gauge potential $A_{\lambda}(x)$ is continuous inside each hypercube and, on the intersection of two neighboring hypercubes $x \in h(m-$ $\widehat{\mu}) \cap h(m)$, the component transverse to this intersection (namely, $\lambda \neq \mu$ ) is continuous across this intersection. We need this property because otherwise boundary terms arising from integration by parts are not cancelled in the following expression. It is easy to see this property if one notes that Lüscher's transition function [40] and the interpolated section [34] are related by

$$
v_{m, \mu}(x)=w^{m-\widehat{\mu}}(x) w^{m}(x)^{-1}, \quad \text { for } \quad x \in h(m-\widehat{\mu}) \cap h(m)
$$

Then from eqs. (7.39) and (7.44) one infers that

$$
w^{m-\widehat{\mu}}(x)^{-1}\left[\partial_{\lambda}+A_{\lambda}^{(m-\widehat{\mu})}(x)\right] w^{m-\widehat{\mu}}(x)=w^{m}(x)^{-1}\left[\partial_{\lambda}+A_{\lambda}^{(m)}(x)\right] w^{m}(x),
$$

for $x \in h(m-\widehat{\mu}) \cap h(m)$. Namely, the interpolated gauge potentials defined from a side of the hypercube $h(m-\widehat{\mu})$ and defined from a side of $h(m)$ coincide on the intersection when $\lambda \neq \mu$. For $\lambda=\mu$, the component may jump across the intersection [34], but this causes no problem for our purpose. The interpolation for the ghost field is obtained by setting $g(n)=\exp [\lambda c(n)]$ in the interpolation formula for the gauge transformation $g(x)$ in ref. [34]. This gives the smooth ghost field (which is also Lie algebra valued) throughout the whole lattice.

(iii) The smoothness and locality. The interpolated gauge potential $A_{\lambda}(x)$ and the ghost field $c(x)$ are smooth functions of link variables (and of the gauge transformation function) residing nearby the point $x$. The smoothness (for the perturbative configurations) and the locality are manifest from the explicit expressions for $A_{\lambda}^{(m)}(x)$ and for $g(x)$ in ref. [34]. In fact, in this case, the relation is ultra-local.

(iv) The correct continuum limit. In the classical continuum limit, $a \rightarrow 0$, the interpolated gauge potential $A_{\mu}(x)$ and the ghost field $c(x)$ reduce (for smooth configurations) to the gauge potential and the ghost field in the continuum theory. From eq. (7.40), we have

$$
\begin{aligned}
\mathcal{P} \exp \left[\int_{0}^{1} d u A_{\mu}(n+(1-u) \widehat{\mu})\right] & =w^{m}(n)^{-1} u_{n, n+\widehat{\mu}^{m}}^{m}(n+\widehat{\mu}) \\
& =U(n, \mu),
\end{aligned}
$$


where we have used the definition of the link variable in the complete axial gauge $u_{n, n+\widehat{\mu}}^{m}[40]^{\S}$. This is nothing but the conventional expression that one assumes in the classical continuum limit, eq. (2.21). For the interpolated ghost field, the formula in ref. [34] shows that $c(x=$ $n)=c(n)$.

(v) The constant ghost field. From the formula in ref. [34], it is easy to see that the constant ghost field on the sites induces the constant interpolated ghost field,

$$
c(n)=c=\text { const. } \Rightarrow c(x)=c=\text { const. }
$$

Now we can write down the nontrivial local solution to the consistency condition (1.3) which satisfies eq. (7.22) and the assumptions (I) and (II) in terms of the interpolated fields. It is given by

$$
\begin{aligned}
\mathcal{A}=-\frac{\epsilon_{H}}{24 \pi^{2}} \sum_{n} \int_{h(n)} & d^{4} x\left\{\varepsilon_{\mu \nu \rho \sigma} \operatorname{tr} c^{(\alpha)}(x) \partial_{\mu}\left[A_{\nu}^{(\alpha)}(x) \partial_{\rho} A_{\sigma}^{(\alpha)}(x)+\frac{1}{2} A_{\nu}^{(\alpha)}(x) A_{\rho}^{(\alpha)}(x) A_{\sigma}^{(\alpha)}(x)\right]\right. \\
& +\varepsilon_{\mu \nu \rho \sigma} c^{U(1)_{\beta}}(x) \partial_{\mu} A_{\nu}^{U(1)_{\beta}}(x) \partial_{\rho} A_{\sigma}^{U(1)_{\beta}}(x) \\
& +\varepsilon_{\mu \nu \rho \sigma} c^{U(1)_{\beta}}(x) \operatorname{tr} \partial_{\mu}\left[A_{\nu}^{(\alpha)}(x) \partial_{\rho} A_{\sigma}^{(\alpha)}(x)+\frac{2}{3} A_{\nu}^{(\alpha)}(x) A_{\rho}^{(\alpha)}(x) A_{\sigma}^{(\alpha)}(x)\right] \\
& \left.+2 \varepsilon_{\mu \nu \rho \sigma} \operatorname{tr}\left[c^{(\alpha)}(x) \partial_{\mu} A_{\nu}^{(\alpha)}(x)\right] \partial_{\rho} A_{\sigma}^{U(1)_{\beta}}(x)\right\} .
\end{aligned}
$$

In this expression, $h(n)$ is the hypercube whose origin is the site $n$. Note that this is a functional of the link variable $U(n, \mu)$ and the ghost field $c(n)$, through the interpolation formulas of ref. [34].

It is easy to see that eq. (7.50) satisfies the consistency condition (1.3), because the BRS transformation of the interpolated fields (7.45) has an identical form as that of the continuum theory and eq. (7.50) has formally an identical form as the gauge anomaly in the continuum theory (2.28). More precisely, we need to perform an integration by parts within each hypercube to show eq. (1.3). Then the transverse continuity (ii) guarantees that contributions from a boundary of hypercubes cancel each other. This solution eq. (7.50) is moreover $\delta_{B}$-nontrivial: From the property (iv) of the interpolation, in the classical continuum limit

$\S$ In fact, from the formulas of ref. [34], it can be seen that $A_{\mu}(x)$ is constant along the link, $A_{\mu}(x)=A_{\mu}(n)$ for $x \in[n, n+\widehat{\mu}]$ where $U(n, \mu)=\exp A_{\mu}(n)$. 
(assuming background fields are smooth), eq. (7.50) reproduces the gauge anomaly in the continuum theory (2.28) which is BRS nontrivial. In other words, if eq. (7.50) is $\delta_{B}$-trivial, there exists a local functional $\mathcal{B}$ on lattice such that $\mathcal{A}=\delta_{B} \mathcal{B}$. Then the classical continuum limit of $\mathcal{B}$, which is a local functional in the continuum theory, cancels the gauge anomaly in the continuum theory.

The nontrivial solution (7.50) manifestly fulfills the condition (7.22) from the properties (v) and (ii) of the interpolation. From the above arguments, it is also clear that eq. (7.50) satisfies the assumption (I) (within the perturbative region (2.9)) and (II).

The existence of the nontrivial solution $\mathcal{A}$ (7.50), which satisfies eq. (7.22) and the assumptions (I) and (II), shows the existence of the unique nontrivial sequence $\widetilde{\mathcal{A}}_{\ell}(7.23)$. The expansion of $\mathcal{A}(7.50)$ in powers of the gauge potential (2.8) gives the unique sequence $\widetilde{\mathcal{A}}_{\ell}$. Note that when the anomaly in the continuum is canceled, the anomaly $\mathcal{A}$ (7.50) vanishes.

Therefore the above procedure gives $\widetilde{\mathcal{A}}_{\ell}=0$ for all $\ell$. This is consistent with the conclusion in the preceding subsection.

\section{Conclusion}

In this paper, we have studied the gauge anomaly $\mathcal{A}$ defined on a 4-dimensional infinite lattice while keeping the lattice spacing finite. We assumed that (I) $\mathcal{A}$ depends smoothly and locally on the gauge potential, (II) $\mathcal{A}$ reproduces the gauge anomaly in the continuum theory, and (III) $U(1)$ gauge anomalies have the topological property. We have then shown that $\mathcal{A}$ can always be removed by local counterterms order by order in powers of the gauge potential: The unique exception is proportional to the anomaly in the continuum theory. This implies that the anomaly cancellation condition in lattice gauge theory is identical to that of the continuum theory.

As we have shown, the gauge anomaly in the formulation based on the Ginsparg-Wilson Dirac operator satisfies the necessary prerequisites for our result (at least for a particular choice of the integration measure) and thus our theorems are applicable (at least in the perturbative region in which a parameterization of the admissible space in terms of the gauge potential is possible). Unfortunately, the gauge anomaly $\mathcal{A}$ appearing in formulations based on the more familiar Wilson Dirac operator or on the Kogut-Susskind Dirac operator is not local, although these Dirac operators themselves are ultra-local. For these operators, the 
chiral gauge symmetry is broken at tree level, and as a result the anomaly is given as $\mathcal{A}=$ $\operatorname{Tr}($ explicit breaking term $) \times$ (propagator). The (massless) propagator in this expression breaks the locality. (In the classical continuum limit, locality is restored and $\mathcal{A}$ reproduces the gauge anomaly in the continuum theory $[63,64]$.)

Let us discuss possible extensions of the results in this paper. The most severe limitation of our result for nonabelian theories is that it holds only in an expansion in powers of the gauge potential. An interesting observation related to this is that the expansion of the anomaly density $a(n)\left(\mathcal{A}=\sum_{n} a(n)\right) a(n)=\sum_{\ell=1}^{\infty} a_{\ell}(n)$ in powers of the gauge potential has a finite radius of convergence. This follows from the smoothness and the locality of the anomaly $\mathcal{A}$ which we have assumed. If these hold for the admissible configurations (2.1), the radius of convergence of this series is given by the right hand side of eq. (2.9). Another interesting point is that the compact solution (7.50) is smooth and local at least in the perturbative region (2.9). These observations suggest that our result is valid beyond the expansion with respect to the gauge potential, at least within the perturbative region. What is not clear at present is a convergence of the individual series $\widetilde{\mathcal{A}}=\sum_{\ell=1}^{\infty} \widetilde{\mathcal{A}}_{\ell}$ and $\mathcal{B}=\sum_{\ell=1}^{\infty} \mathcal{B}_{\ell}$ in eq. (7.23).

By using similar arguments as above, it seems straightforward to classify general topological fields on a 4-dimensional infinite lattice (which is a nonabelian analogue of the theorem (5.56)) at least to all orders in powers of the gauge potential. According to the result of ref. [21], this analysis is relevant for the existence of an exactly gauge invariant formulation of anomaly-free two-dimensional chiral gauge theories. For four-dimensional chiral gauge theories, we have to generalize the covariant Poincaré lemma (5.11) to a 6-dimensional lattice. This generalization would be straightforward, although the proof may become quite cumbersome.

The restriction to the perturbative region (2.9) for nonabelian theories is due to a complicated structure of the admissible space (2.1). If it is possible to parameterize the admissible space in terms of the gauge potential, ${ }^{\star}$ as in the abelian case, the restriction may be relaxed. It is highly plausible that the "rewinding" technique of ref. [35] is useful in this context.

Our results are not yet "realistic" because these are for infinite lattice size. For the abelian case $G=U(1)$, it has been shown [20] that the anomaly cancellation works even

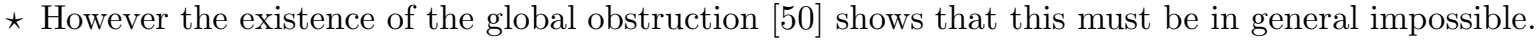


for a finite periodic lattice. To generalize the argument in ref. [20] for nonabelian theories, we have to understand first the structure of the admissible space on a finite lattice (see the above remark). On the other hand, another reason why our proof is valid only for an infinite lattice is that our proof of the algebraic Poincaré lemma (5.1) relys on the Poincaré lemma of ref. [19], which guarantees the triviality of the de Rham cohomology on an infinite lattice. Physically, one expects that the $d$-cohomology on local functions of gauge and ghost fields is independent on the possibly nontrivial de Rham cohomology on a finite lattice, because the dependence is local. It is thus highly desirable to show the algebraic Poincaré lemma in a way being independent of the de Rham cohomology. In the continuum theory, this is actually possible $[16,17]$.

In this paper, we adopted a "classical" algebraic viewpoint based on the Wess-Zumino consistency condition. In the continuum theory, the algebraic approach to the anomaly has a close relationship to a higher dimensional theory [65]. It seems very important to investigate such a relationship in the context of lattice gauge theory. In fact, there are some indications that such a relation exists $[21,45,66]$.

Finally, let us remark on the physical implications of these analyses. After all, even if a local counterterm which makes the effective action gauge invariant exists (for anomaly-free cases), the implementation of gauge invariance requires a fine tuning of parameters in the counterterm which is highly unnatural. One might thus be tempted to apply the mechanism of ref. [67] which dynamically restores the gauge invariance. However, for the mechanism of ref. [67] to work, the gauge breaking $\mathcal{A}$ (with the ghost field is replaced by a logarithm of the gauge transformation field) has to be "small." In particular, if $\mathcal{A} \neq \delta_{B} \mathcal{B}$ for a local functional $\mathcal{B}$, the effective lagrangian for the gauge transformation field is given by a lattice analogue of the Wess-Zumino lagrangian which modifies the physical content (thus it cannot be regarded as "small"). Therefore, the study of the gauge anomaly on lattice is important also in order to examine the necessary condition for the mechanism of ref. [67]. In this respect, it seems interesting to study the locality (in a four dimensional sense) of the gauge anomaly appearing in the overlap formulation with the Brillouin-Wigner phase convention, in connection with the result of ref. [68].

The author has greatly benefited from correspondence with T. Fujiwara, Y. Kikukawa and $\mathrm{K}$. Wu and from discussions with P. Hernández. He is particularly grateful to M. Lüscher for various helpful remarks, without which this work would not have been completed. The 
author is also indebted to A. Grassi and to Y. Shamir who independently explained to him that the statement concerning $U(1)$ gauge anomalies, which was made in the previous version of this paper, was not correct in general.

\section{APPENDIX A}

Here we summarize our notation and the convention. Throughout this paper, we consider the 4-dimensional infinite lattice $Z^{4}$. The sites of the lattice are denoted by $n, m$, etc. The lattice spacing is taken to be unity $a=1$ unless otherwise stated. The Greek letters $\mu$, $\nu$, etc. denote the Lorentz indices which run from 1 to 4 . $\widehat{\mu}$ stands for the unit vector in direction $\mu$. For Lorenz indices, the summation over repeated indices is always understood. The Levi-Civita symbol is defined by $\varepsilon_{\mu \nu \rho \sigma}=\varepsilon_{[\mu \nu \rho \sigma]}$ and $\varepsilon_{1234}=1$.

The forward and the backward difference operators are respectively defined by

$$
\Delta_{\mu} f(n)=f(n+\widehat{\mu})-f(n), \quad \Delta_{\mu}^{*} f(n)=f(n)-f(n-\widehat{\mu}) .
$$

The symbol $\partial_{\mu}$ is reserved for the standard derivative.

$H=R$ or $L$ stands for the chirality of a Weyl fermion, and we set $\epsilon_{R}=+1$ and $\epsilon_{L}=-1$.

$G=\prod_{\alpha} G_{\alpha}$ is the (compact) gauge group where $G_{\alpha}$ denotes a simple group or a $U(1)$ factor. The Greek indices $\alpha, \beta$, etc. are used to label each factor group. $T^{a}$ stands for the representation matrix of the Lie algebra, $\left[T^{a}, T^{b}\right]=i f^{a b c} T^{c}$. The summation over repeated group indices $a, b$, etc. is always understood.

$U(n, \mu)$ is the link variable on the link that connects the lattice sites $n$ and $n+\widehat{\mu}$. For the abelian gauge group $G=U(1)^{N}$, we parameterize the link variable by the gauge potential as $U^{a}(n, \mu)=\exp A_{\mu}^{a}(n)$. In this case, the superscript $a$ distinguishes each $U(1)$ factor. The abelian field strength is defined by

$$
F_{\mu \nu}^{a}(n)=\Delta_{\mu} A_{\nu}^{a}(n)-\Delta_{\nu} A_{\mu}^{a}(n)
$$

We never use this symbol $F_{\mu \nu}^{a}$ to indicate the nonabelian field strength. The plaquette variable is defined by

$$
P(n, \mu, \nu)=U(n, \mu) U(n+\widehat{\mu}, \nu) U(n+\widehat{\nu}, \mu)^{-1} U(n, \nu)^{-1}
$$




\section{APPENDIX B}

In this appendix, we show the calculation of the "Wilson line" $W^{\prime}$ in eq. (2.17). From eqs. (2.11) and (2.17), $W^{\prime}$ is given by

$$
W^{\prime}=\exp \left[\epsilon_{H} \int_{0}^{1} d t \int_{0}^{1} d s \operatorname{Tr} P_{t}(s)\left[\partial_{s} P_{t}(s), \partial_{t} P_{t}(s)\right]\right]
$$

where $P_{t}(s)=\left.P_{H}\right|_{U \rightarrow U_{t}(s)}$ and we explicitly indicated $s$-dependences defined through eq. (2.13). If we introduce the transporting operator $Q_{t}(s)$ for each $s$ by $\partial_{t} Q_{t}(s)=\left[\partial_{t} P_{t}(s), P_{t}(s)\right] Q_{t}(s)$ and $Q_{0}(s)=1$, we have

$$
P_{t}(s)=Q_{t}(s) P_{0}(s) Q_{t}(s)^{\dagger}
$$

(note that $Q_{t}(s)$ is unitary). Substituting this into eq. (B.1), and after some calculation, we have

$$
W^{\prime}=\exp \left\{\epsilon_{H} \int_{0}^{1} d t \int_{0}^{1} d s\left[\partial_{s} \operatorname{Tr} P_{0}(s) Q_{t}^{\dagger}(s) \partial_{t} Q_{t}(s)-\partial_{t} \operatorname{Tr} P_{0}(s) Q_{t}^{\dagger}(s) \partial_{s} Q_{t}(s)\right]\right\}
$$

We then apply the Stokes theorem to this 2-dimensional integration. This yields

$$
W^{\prime}=\exp \left[\epsilon_{H} \int_{0}^{1} d t \operatorname{Tr} P_{0}(1) Q_{t}^{\dagger}(1) \partial_{t} Q_{t}(1)-\left.\epsilon_{H} \int_{0}^{1} d s \operatorname{Tr} P_{0}(s) Q_{t}^{\dagger}(s) \partial_{s} Q_{t}(s)\right|_{t=0} ^{t=1}\right]
$$

However, from eq. (B.2),

$$
\operatorname{Tr} P_{0}(1) Q_{t}^{\dagger}(1) \partial_{t} Q_{t}(1)=\operatorname{Tr} P_{0}(1) Q_{t}^{\dagger}(1)\left[\partial_{t} P_{t}(1), P_{t}(1)\right] Q_{t}(1)=0
$$

and $\partial_{s} Q_{0}(s)=0$ because $Q_{0}(s)=1$. Therefore

$$
W^{\prime}=\exp \left[-\epsilon_{H} \int_{0}^{1} d s \operatorname{Tr} P_{0}(s) Q_{1}^{\dagger}(s) \partial_{s} Q_{1}(s)\right]
$$

Noting that $P_{0}(s) \partial_{s} P_{0}(s) P_{0}(s)=0$ and $P_{1}(s)=P_{0}(s)$ (recall that $U_{1}(s)=U_{0}(s)$ ), it can be confirmed that eq. (B.6) is equal to

$$
W^{\prime}=\exp \left\{-\epsilon_{H} \int_{0}^{1} d s \operatorname{Tr}\left[1-P_{0}(s)+P_{0}(s) Q_{1}(s)\right]^{-1} \partial_{s}\left[1-P_{0}(s)+P_{0}(s) Q_{1}(s)\right]\right\}
$$

This proves eq. (2.17). 


\section{REFERENCES}

1. H. B. Nielsen and M. Ninomiya, Phys. Lett. B105 (1981) 219; Nucl. Phys. B185 (1981) 20; B195 (1982) 541 (E); B193 (1981) 173.

2. D. Friedan, Commun. Math. Phys. 85 (1982) 481.

3. S. L. Adler, Phys. Rev. 177 (1969) 2426.

4. J. S. Bell and R. Jackiw, Nuovo Cim. 60A (1969) 47.

5. W. A. Bardeen, Phys. Rev. 184 (1969) 1848.

6. C. Bouchiat, J. Iliopoulos and Ph. Meyer, Phys. Lett. 38B (1972) 519.

7. H. Georgi and S. Glashow, Phys. Rev. D6 (1972) 429.

8. D. Gross and R. Jackiw, Phys. Rev. D6 (1972) 477.

9. For a review on various approaches, Y. Shamir, Nucl. Phys. Proc. Suppl. 47 (1996) 212.

10. C. Becchi, A. Rouet and R. Stora, Comm. Math. Phys. 42 (1975) 127; Ann. Phys. 98 (1976) 287.

11. R. Stora, in New developments in quantum field theory and statistical mechanics (Cargèse 1976), eds. M. Lévy and P. Mitter, (Plenum Press, New York, 1977).

12. L. Bonora and P. Cotta-Ramusino, Phys. Lett. 107B (1981) 87; Commun. Math. Phys. 87 (1983) 589.

13. R. Stora, in Progress in gauge field theory (Cargèse 1983), eds. G. 't Hooft et al, (Plenum Press, New York, 1984).

14. B. Zumino, in Relativity, groups and topology (Les Houches 1983), eds. B. S. De Witt and R. Stora, (North-Holland, Amsterdam, 1984).

B. Zumino, Y. S. Wu and A. Zee, Nucl. Phys. B239 (1984) 477.

15. L. Baulieu, in Particles and fields (Cargèse 1983), eds. M. Lévy et al, (Plenum Press, New York, 1985); Nucl. Phys. B241 (1984) 557; Phys. Rep. 129 (1985) 1.

16. F. Brandt, N. Dragon and M. Kreuzer, Phys. Lett. B231 (1989) 263; Nucl. Phys. B332 (1990) 224; B332 (1990) 250.

N. Dragon, Lectures given at Saalburg Summer School (1995), hep-th/9602163. 
17. M. Dubois-Violette, M. Henneaux, M. Talon and C.-M. Viallet, Phys. Lett. B267 (1991) 81; B289 (1992) 361.

18. A. Borrelli, L. Maiani, G. C. Rossi, R. Sisto and M. Testa, Phys. Lett. B221 (1989) 360; Nucl. Phys. B333 (1990) 335.

19. M. Lüscher, Nucl. Phys. B538 (1999) 515.

20. M. Lüscher, Nucl. Phys. B549 (1999) 295.

21. M. Lüscher, Nucl. Phys. B568 (2000) 162; for a review, Nucl. Phys. Proc. Suppl. 83-84 (2000) 34 .

22. J. Wess and B. Zumino, Phys. Lett. 37B (1971) 95.

23. P. H. Ginsparg and K. G. Wilson, Phys. Rev. D25 (1982) 2649.

24. P. Hasenfratz, Nucl. Phys. Proc. Suppl. 63A-C (1998) 53; Nucl. Phys. B525 (1998) 401.

25. H. Neuberger, Phys. Lett. B417 (1998) 141; B427 (1998) 353.

26. R. Narayanan and H. Neuberger, Phys. Rev. Lett. 71 (1993) 3251; Nucl. Phys. B412 (1994) 574; B443 (1995) 305.

27. S. Randjbar-Daemi and J. Strathdee, Phys. Lett. B348 (1995) 543; Nucl. Phys. B443 (1995) 386; B466 (1996) 335; Phys. Lett. B402 (1997) 134.

28. A. Connes, Noncommutative geometry (Academic Press, New York, 1994).

29. A. Sitarz, J. Geom. Phys. 15 (1995) 123.

30. A. Dimakis and F. Müller-Hoissen, Phys. Lett. B295 (1992) 242; J. Phys. A: Math. Gen. 27 (1994) 3159; J. Math. Phys. 35 (1994) 6703.

A. Dimakis, F. Müller-Hoissen and T. Striker, Phys. Lett. B300 (1993) 141; J. Phys. A: Math. Gen. 26 (1993) 1927.

31. H. G. Ding, H. Y. Guo, J. M. Li and K. Wu, Z. Phys. C64 (1994) 521; J. Phys. A: Math. Gen. 27 (1994) L75; 27 (1994) L231; Commun. Theor. Phys. 21 (1994) 85.

H. Y. Guo, K. Wu and W. Zhang, "Noncommutative differential calculus on discrete abelian groups and its applications," ITP-Preprint, March, 1999.

32. T. Fujiwara, H. Suzuki and K. Wu, Nucl. Phys. B569 (2000) 643. 
33. T. Fujiwara, H. Suzuki and K. Wu, Phys. Lett. B463 (1999) 63; hep-lat/9910030.

34. M. Göckeler, A. S. Kronfeld, M. L. Laursen, G. Schierholz and U.-J. Wiese, Nucl. Phys. B292 (1987) 349.

M. Göckeler, A. S. Kronfeld, G. Schierholz and U.-J. Wiese, Nucl. Phys. B404 (1993) 839.

35. P. Hernández and R. Sundrum, Nucl. Phys. B455 (1995) 287; B472 (1996) 334.

36. M. Reed and B. Simon, Functional analysis (Academic Press, New York, 1972).

37. P. Hernández, K. Jansen and M. Lüscher, Nucl. Phys. B552 (1999) 363.

38. P. Hasenfratz, V. Laliena and F. Niedermayer, Phys. Lett. B427 (1998) 125.

39. M. Lüscher, Phys. Lett. B428 (1998) 342.

40. M. Lüscher, Commun. Math. Phys. 85 (1982) 39.

41. F. Niedermayer, Nucl. Phys. Proc. Suppl. 73 (1999) 105.

42. I. Horvath, Phys. Rev. Lett. 81 (1998) 4063; Phys. Rev. D60 (1999) 034510.

43. W. Bietenholz, hep-lat/9901005.

44. T. Fujiwara, H. Suzuki and K. Wu, hep-lat/0001029.

45. T. Aoyama and Y. Kikukawa, hep-lat/9905003.

46. H. Neuberger, hep-lat/9912013.

47. R. Narayanan, Phys. Rev. D58 (1998) 097501.

48. Y. Kikukawa and A. Yamada, Nucl. Phys. B547 (1999) 413.

49. H. Suzuki, Prog. Theor. Phys. 101 (1999) 1147.

50. O. Bär and I. Campos, Nucl. Phys. Proc. Suppl. 83-84 (2000) 594; hep-lat/0001025, to appear in Nucl. Phys. B.

51. E. Witten, Phys. Lett. 117B (1982) 324.

52. K. Fujikawa, Nucl. Phys. B546 (1999) 480.

53. Y. Kikukawa and A. Yamada, Phys. Lett. B448 (1999) 265.

54. D. H. Adams, hep-lat/9812003. 
55. H. Suzuki, Prog. Theor. Phys. 102 (1999) 141.

56. W. Bardeen and B. Zumino, Nucl. Phys. B244 (1984) 421.

57. H. Neuberger, Phys. Lett. B437 (1998) 117; Phys. Rev. D59 (1999) 085006.

58. S. Ferrara, O. Piguet and S. Schweda, Nucl. Phys. B119 (1977) 493.

59. K. Fujikawa, Prog. Theor. Phys. 59 (1978) 2045.

60. L. Bonora and M. Tonin, Phys. Lett. 98B (1981) 48.

61. L. D. Faddeev, Phys. Lett. 145B (1984) 81.

62. See, for example, L. O'Raifeartaigh, Group structure of gauge theories (Cambridge University Press, Cambridge, 1986).

63. S. Aoki, Phys. Rev. D35 (1986) 1435.

64. A. Coste, C. Korthals Altes and O. Napoly, Phys. Lett. 179B (1986) 125; Nucl. Phys. B289 (1987) 645.

65. L. Alvarez-Gaume and P. Ginsparg, Nucl. Phys. B243 (1984) 449.

66. D. H. Adams, hep-lat/9910036; hep-lat/0001014.

67. D. Foerster, H. B. Nielsen and M. Ninomiya, Phys. Lett. 94B (1980) 135.

68. M. Golterman and Y. Shamir, Phys. Lett. B353 (1995) 84; B359 (1995) 422 (E); Nucl. Phys. Proc. Suppl. 47 (1996) 603. 